Understanding and predicting stiffness in advanced fibre-reinforced polymer (FRP) composites for structural applications

R. M. GUEDES, University of Porto, Portugal and J. XA VIER, CITAB/UTAD, Portugal

DOI: $10.1533 / 9780857098641.3 .298$

\begin{abstract}
This chapter describes the elastic qualities of advanced fibrereinforced composites, in terms of characterization, measurement and prediction from the basic constituents, i.e. the fibre and matrix. The elastic analysis comprises applying micromechanics approaches to predict the lamina elastic properties from the basic constituents, and using classical lamination theory to predict the elastic properties of composite materials composed of several laminae stacked at different orientations. Examples are given to illustrate the theoretical analysis and give a full apprehension of its prediction capability. The last section provides an overview on identification methods for elastic proprieties based on full-field measurements. It is shown that these methodologies are very convenient for elastic characterization of anisotropic and heterogeneous materials.
\end{abstract}

Key words: elastic modulus, orthotropic, micromechanics, classical lamination theory, digital image correlation, optical methods.

\title{
11.1 Introduction
}

Polymer matrix-based composites are essentially composed of fibres embedded in polymeric matrices. Polymeric matrices are divided into thermoplastic and thermoset resins. The fibres play an important role by their reinforcing character, enhancing the mechanical performance of these composites to high levels. These arrangements are more than simple combinations of fibres and polymeric matrices, since the synergistic effects are important in their global mechanical performance. In the case of continuous reinforcement, fibres are disposed mainly on the surface ply, either unidirectional or woven fabric, resulting in superior mechanical properties in the ply surface directions. Consequently, in the thickness direction the mechanical properties of composites are low. The complexity of these systems poses some challenges on the models used to predict stiffness evolution, when general loading conditions are applied, including the environmental effects.

The reinforcements can have different geometries - particulate, flake or fibres. The fibres can be continuous, discontinuous and oriented, or randomly 
distributed (see Fig. 11.1). The polymer matrices have much lower stiffness compared to the reinforcements. The matrix holds the reinforcements, protecting them against environmental and external aggression. Simultaneously the matrix allows an effective stress transfer to the reinforcements. This relies on good adhesion between the matrix and the reinforcement. The mechanical performance of fibre-reinforced polymers depends on different factors such as fibre length, fibre orientation and fibre shape. The circular shape is more usual since it is easier to produce, but other shapes are possible.

This chapter is devoted to the analysis of the elastic properties and their characterization for laminated advanced composites. It starts with a general overview of composite stiffness and then moves to lamina analysis, focused on unidirectional reinforced composites. The analysis of laminated composites is addressed through the classical lamination theory (CLT). The last section describes full-field techniques coupled with inverse identification methods that can be employed to measure the elastic constants.

\subsection{General aspects of composite stiffness}

Composite materials consist of two or more constituents mixed at a nano-, micro- or macroscopic level. These constituents are not soluble and form distinct phases. The reinforcing phase is embedded in the other phase, designated the matrix. Usually the reinforcing material is in the form of continuous or short fibres or particles. Actually, composites provide a more efficient way for using materials in structural applications. For example, they allow mass reduction without decreasing the stiffness and strength of components, by

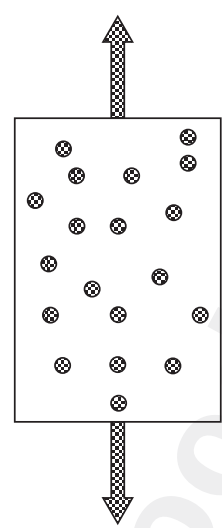

(a)

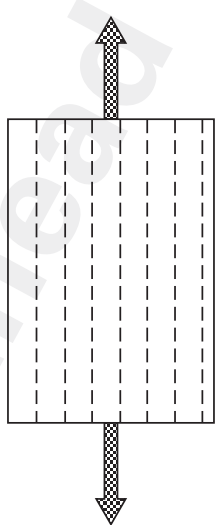

(b)

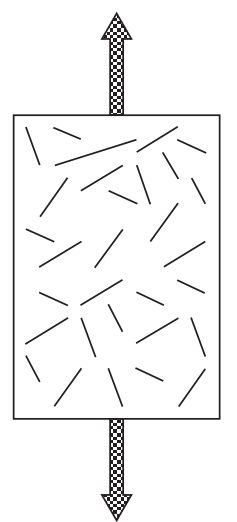

(c)

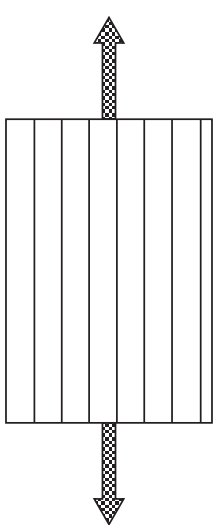

(d)

11.1 (a) Particulate, (b) oriented discontinuous fibres, (c) randomly distributed discontinuous fibres, (d) continuous fibres. 
replacing conventional metal alloys with composite materials. The reduction in weight and number of parts in an assembly largely compensates for the higher composite material costs. Moreover, composite systems often present improved fatigue, impact resistance, thermal conductivity and corrosion resistance.

The lightest structural component for a specified deformation, i.e. strain or deflection, under a specified load is the one with the highest specific modulus. This is calculated as the ratio of the Young's modulus to the density of the material. For comparison purposes in Fig. 11.2 is plotted the specific modulus for several materials, including typical reinforcing fibres. Carbon-reinforced composites possess higher specific modulus when compared against aluminium and steel [1].

The stiffness of polymer-based composite systems depends on numerous factors such as the stiffness of constituents, the volume fraction of each component, and the size, shape and orientation of reinforcements. As a whole there are three distinct types of polymer composites: continuous fibrereinforced polymer composites, short fibre-reinforced polymer composites, and polymer nanocomposites. Theoretical models based on micromechanical models are well developed and provide an adequate representation of composite stiffness. These micromechanical models are formulated based on assumptions of continuum mechanics. However, for nanocomposite materials, with fillers of size approximately $1 \mathrm{~nm}$ compared to the typical carbon fibre diameter of $50 \mu \mathrm{m}$, the rules and requirements for continuum

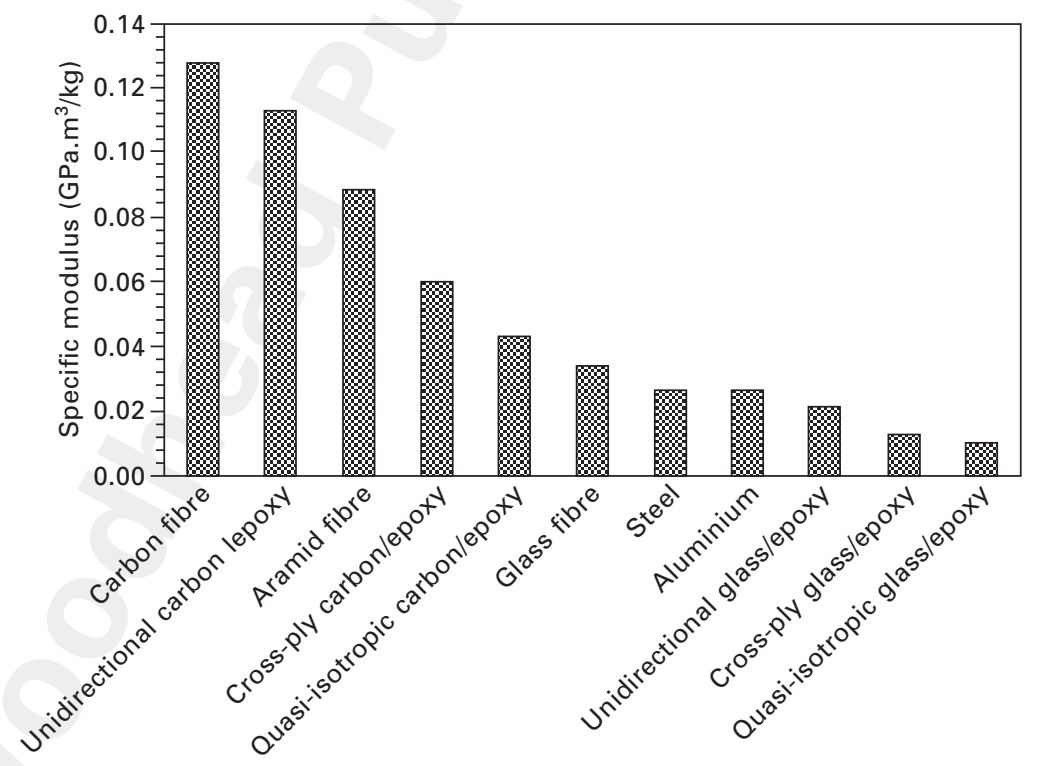

11.2 Specific modulus of different materials.

(c) Woodhead Publishing Limited, 2013 
modelling are not satisfied. Several studies have attempted to address the applicability of continuum micromechanics to nano-reinforced polymer composites, by taking into account the effects associated with the significant size difference between nano fillers and typical carbon fibre. Nonetheless the classical approach can capture the main effects of nanoparticles on the elastic properties of nanocomposites. Particle clustering is the phenomenon mainly responsible for deviations of experimental data from theoretical predictions.

Since the reinforcements possess higher modulus and strength than the polymer matrix, internal mismatches are induced, leading to local stress concentrations. Depending on the loading direction, fibre-aligned cracks may start to form. In the literature the terms matrix microcracks, microcracks, intralaminar cracks, ply cracks and transverse cracks often describe the same phenomenon [2]. These are observed during tensile loading, fatigue loading and thermocycling. Microcracks appear predominantly in plies off-axis to loading directions. This phenomenon leads to degradation in all effective moduli, Poisson's ratios, thermal expansion coefficients [3] and moisture diffusion coefficients [3, 4]. Furthermore, the nucleation of microcracks may induce delamination [2].

The application of damage mechanics enables one to model matrix degradation in fibre-reinforced polymers due to cracking [5]. This damage is quantified in terms of crack density, i.e. the reciprocal of crack spacing. The model used by Roberts et al. [6], based on the previous work of Zang and Gudmundson [7,8], applies to microcracks with crack surfaces parallel to the fibre direction and perpendicular to the lamina plane.

The viscoelastic and viscoplastic nature of polymeric materials that constitutes the composite matrix makes their mechanical behaviour timeor rate-dependent. Polymers are also temperature- and moisture-dependent displaying, in certain cases, large stiffness variations. Physical ageing is also an important issue that is often ignored for simplification purposes. A recent overview concerning these important matters is given elsewhere [9].

\subsection{Understanding lamina stiffness}

The typical building block of a composite structure is the lamina, with a typical thickness of $0.125 \mathrm{~mm}$. The lamina stress-strain relationships are described for orthotropic, transverse isotropic and isotropic materials. When a lamina is reinforced with unidirectional fibres it can be assumed to be a transversely isotropic material. In this chapter, theoretical determination of lamina elastic properties, assumed to be a transversely isotropic material, using micromechanics approaches is presented and illustrated with experimental data. 


\subsubsection{The representative volume element (RVE)}

Knowledge of the exact configuration of fibres and matrix in a composite structure is impractical for structural analysis. Observation of a typical micrograph of a cross-section of a unidirectional glass or carbon/epoxy lamina makes it clear that the fibre distribution is not uniform but is fairly random [10]. This provokes irregular gaps and contiguity, i.e. fibres touching in some locations. Similar cross-sections can be computationally randomly generated as shown in Fig. 11.3. Detailed discussion on this subject can be found elsewhere [11-13].

The effective properties of a composite material correspond to properties averaged over a repeating representative volume element (RVE). This element should be large enough to represent the microstructure yet sufficiently smaller than the macroscopic structural dimensions. In fibre-reinforced composites, the RVE length scale is several times the fibre diameter. If the RVE dimension is small compared with the characteristic dimensions of the structure, the material can be assumed as homogeneous. Figure 11.4 depicts a schematic example of a RVE with other examples of elements that cannot be considered RVEs.

\subsubsection{Generalized Hooke's law}

The most general linear elastic relationship between the stresses and strains is given as

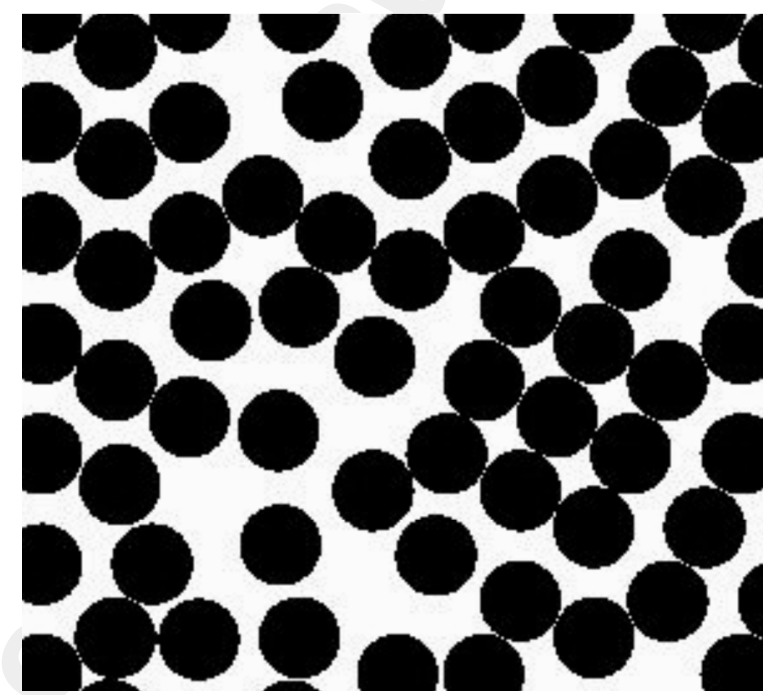

11.3 Example of randomly distributed fibres computationally generated over a lamina cross-section. 


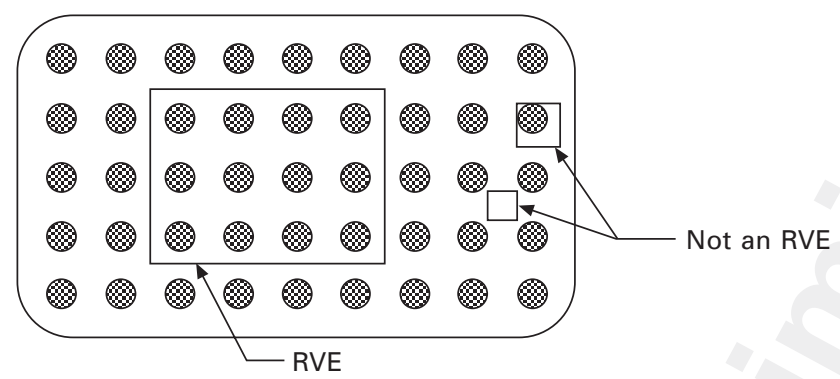

11.4 Example of a representative volume element (adapted from [14]).

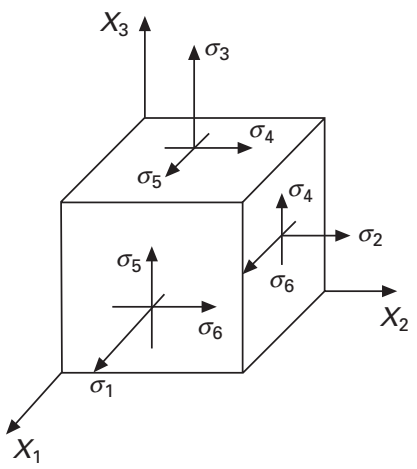

11.5 Three-dimensional representation of the stresses.

$$
\sigma_{i j}=C_{i j k l} \varepsilon_{k l}
$$

where $\sigma_{k l}$ represent the stresses and $\varepsilon_{k l}$ the strains, and $C_{i j k l}$ is a fourthorder tensor with 81 constants called the elastic moduli. This linear elastic stress-strain constitutive law is called the Generalized Hooke's law.

Inverting (11.1) the strains are given in terms of stresses in the form

$$
\varepsilon_{i j}=C_{i j k l}^{-1} \sigma_{k l}
$$

where the compliance $S_{i j k l}=C_{i j k l}^{-1}$ is defined as the inverse of the stiffness.

When the stresses and strains are symmetric the number of independent constants is reduced to 36 . Hooke's law can be written in a contracted notation:

$$
\sigma_{i}=C_{i j} \varepsilon_{j}(i, j=1,2, \ldots, 6)
$$

A three-dimensional representation of the stresses in contract notation is presented in Fig. 11.5.

If there exists a strain density function such as

$$
W=\frac{1}{2} C_{i j} \varepsilon_{i} \varepsilon_{j}
$$


and it verifies

$$
\sigma_{i}=\frac{\partial W}{\partial \varepsilon_{i}}
$$

then $c_{i j}$ is symmetric and the number of independent elastic constants is reduced to 21 . A material with 21 independent constants is called anisotropic. The stiffness matrix is written as

$$
\left[C_{i j}\right]=\left[\begin{array}{cccccc}
C_{11} & C_{12} & C_{13} & C_{14} & C_{15} & C_{16} \\
& C_{22} & C_{23} & C_{24} & C_{25} & C_{26} \\
& & C_{33} & C_{34} & C_{35} & C_{36} \\
& & & C_{44} & C_{45} & C_{46} \\
& & & & C_{55} & C_{56} \\
\text { Sym. } & & & & & C_{66}
\end{array}\right]
$$

The inverted matrix gives the symmetric compliance,

$$
\left[S_{\mathrm{ij}}\right\rfloor=\left[C_{i j}\right]^{-1}=\left[\begin{array}{cccccc}
S_{11} & S_{12} & S_{13} & S_{14} & S_{15} & S_{16} \\
& S_{22} & S_{23} & S_{24} & S_{25} & S_{26} \\
& & S_{33} & S_{34} & S_{35} & S_{36} \\
& & & S_{44} & S_{45} & S_{46} \\
& & & & S_{55} & S_{56} \\
\text { Sym. } & & & & & S_{66}
\end{array}\right]
$$

\subsubsection{Material symmetry: orthotropic, transversely isotropic and isotropic materials}

Consider a material that is symmetric about two planes: the $x_{1}-x_{2}$ and $x_{2}-x_{3}$ planes as shown in Fig. 11.6. It must be expected that the elastic constants in both coordinate systems (unprimed and primed) are identical, i.e. $C_{i j}=C_{i j}^{\prime}$.

The definition of the coordinate systems leads to

$$
\begin{aligned}
& \sigma_{1}=\sigma_{1}^{\prime} \sigma_{4}=-\sigma_{4}^{\prime} \\
& \sigma_{2}=\sigma_{2}^{\prime} \sigma_{5}=-\sigma_{5}^{\prime} \\
& \sigma_{3}=\sigma_{3}^{\prime} \sigma_{6}=-\sigma_{6}^{\prime} \\
& \varepsilon_{1}=\varepsilon_{1}^{\prime} \varepsilon_{4}=-\varepsilon_{4}^{\prime} \\
& \varepsilon_{2}=\varepsilon_{2}^{\prime} \varepsilon_{5}=-\varepsilon_{5}^{\prime} \\
& \varepsilon_{3}=\varepsilon_{3}^{\prime} \varepsilon_{6}=-\varepsilon_{6}^{\prime}
\end{aligned}
$$




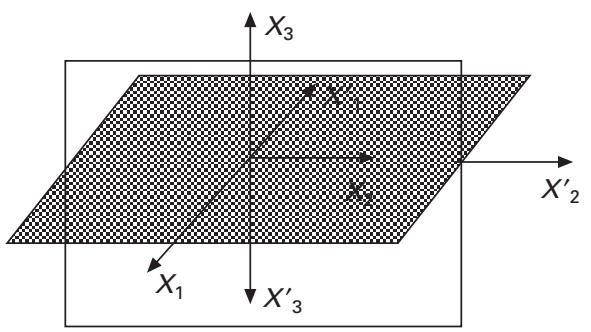

11.6 Symmetry about the $x_{1}-x_{2}$ and $x_{2}-x_{3}$ planes.

Expressing $\sigma_{1}=\sigma_{1}^{\prime}$ in terms of strains and elastic constants using the Generalized Hooke's law,

$$
\begin{aligned}
& C_{11} \varepsilon_{1}+C_{12} \varepsilon_{2}+C_{13} \varepsilon_{3}+C_{14} \varepsilon_{4}+C_{15} \varepsilon_{5}+C_{16} \varepsilon_{6} \\
& =C_{11}^{\prime} \varepsilon_{1}+C_{12}^{\prime} \varepsilon_{2}^{\prime}+C_{13}^{\prime} \varepsilon_{3}^{\prime}+C_{14}^{\prime} \varepsilon_{4}^{\prime}+C_{15}^{\prime} \varepsilon_{5}^{\prime}+C_{16}^{\prime} \varepsilon_{6}^{\prime}
\end{aligned}
$$

Now using the previous relationships, the following is obtained:

$$
C_{14}=C_{15}=C_{16}=0
$$

Using similar procedures, it is possible to conclude that

$$
C_{24}=C_{25}=C_{26}=C_{34}=C_{35}=C_{36}=C_{45}=C_{46}=C_{56}=0
$$

The obtained stiffness matrix corresponds to an orthotropic material with nine independent constants. The constitutive equation can be written in matrix form as

$$
\left\{\begin{array}{c}
\sigma_{1} \\
\sigma_{2} \\
\sigma_{3} \\
\tau_{23} \\
\tau_{13} \\
t_{12}
\end{array}\right\}=\left[\begin{array}{cccccc}
C_{11} & C_{12} & C_{13} & 0 & 0 & 0 \\
& C_{22} & C_{23} & 0 & 0 & 0 \\
& & C_{33} & 0 & 0 & 0 \\
& & & C_{44} & 0 & 0 \\
& & & & C_{55} & 0 \\
\text { Sym. } & & & & & C_{56} \\
\gamma_{23}
\end{array}\right\}
$$

or by inversion

$$
\left\{\begin{array}{c}
\varepsilon_{1} \\
\varepsilon_{2} \\
\varepsilon_{3} \\
\gamma_{23} \\
\gamma_{13} \\
\gamma_{12}
\end{array}\right\}=\left[\begin{array}{cccccc}
S_{11} & S_{12} & S_{13} & 0 & 0 & 0 \\
& S_{22} & S_{23} & 0 & 0 & 0 \\
& & S_{33} & 0 & 0 & 0 \\
& & & S_{44} & 0 & 0 \\
& & & & S_{55} & 0 \\
\text { Sym. } & & & & & S_{56} \\
t_{12}
\end{array}\right\}\left\{\begin{array}{c}
\sigma_{1} \\
\sigma_{2} \\
\sigma_{3} \\
\tau_{23} \\
\tau_{13} \\
t_{12}
\end{array}\right\}
$$


where $\tau_{23}=\sigma_{4}, \tau_{13}=\sigma_{5}, \tau_{12}=\sigma_{6}$ and $\gamma_{23}=\varepsilon_{4}, \gamma_{13}=\varepsilon_{5}, \gamma_{12}=\varepsilon_{6}$.

The stiffness and compliance matrices are described in a very simple manner by the elastic constants. However, the elastic constants are not measured directly. Hence it is important to write the stiffness and compliance matrices as functions of the measured constants called engineering constants.

The compliance matrix for an orthotropic material in terms of engineering constants is given as

$$
\mathbf{S}=\left[\begin{array}{cccccc}
\frac{1}{E_{1}} & -\frac{v_{21}}{E_{2}} & -\frac{v_{31}}{E_{2}} & 0 & 0 & 0 \\
-\frac{v_{12}}{E_{1}} & \frac{1}{E_{2}} & -\frac{v_{32}}{E_{3}} & 0 & 0 & 0 \\
-\frac{v_{31}}{E_{1}} & -\frac{v_{23}}{E_{2}} & \frac{1}{E_{3}} & 0 & 0 & 0 \\
0 & 0 & 0 & \frac{1}{G_{23}} & 0 & 0 \\
0 & 0 & 0 & 0 & \frac{1}{G_{13}} & 0 \\
0 & 0 & 0 & 0 & 0 & \frac{1}{G_{12}}
\end{array}\right]
$$

Since the matrix is symmetric $S_{i j}=S_{j i}$ then it must be true that

$$
\frac{v_{i j}}{E_{i}}=\frac{v_{j i}}{E_{j}}(i, j=1,2,3
$$

The correspondent stiffness matrix is given as

$$
\mathbf{C}=\left[\begin{array}{cccccc}
\frac{1-v_{23} v_{32}}{\Delta} E_{1} & \frac{v_{21}+v_{23} v_{31}}{\Delta} E_{1} & \frac{v_{31}+v_{21} v_{32}}{\Delta} E_{1} & 0 & 0 & 0 \\
\frac{v_{12}+v_{13} v_{32}}{\Delta} E_{2} & \frac{1-v_{31} v_{13}}{\Delta} E_{2} & \frac{v_{32}+v_{12} v_{31}}{\Delta} E_{2} & 0 & 0 & 0 \\
\frac{v_{13}+v_{23} v_{12}}{\Delta} E_{3} & \frac{v_{23}+v_{13} v_{21}}{\Delta} E_{3} & \frac{1-v_{21} v_{12}}{\Delta} E_{3} & 0 & 0 & 0 \\
0 & 0 & 0 & G_{23} & 0 & 0 \\
0 & 0 & 0 & 0 & G_{13} & 0 \\
0 & 0 & 0 & 0 & 0 & G_{12}
\end{array}\right]
$$

where $\Delta=1-\left(v_{31} v_{13}+v_{1} \_+v_{23} v_{32}+v_{23} v_{31} v_{12}+v_{21} v_{13} v_{32}\right)$ 
Figure 11.7 gives an example of an orthotropic fibre-reinforced material where the cross-section of the fibres is oval.

When the cross-section of fibre-reinforced composite is a plane of isotropy, it is called a transversely isotropic material, as described in Fig. 11.8.

Since the elastic properties are isotropic in the plane transverse to the fibres, the plane $x_{2}-x_{3}$ (Fig. 11.8), additional simplifications on elastic constants are obtained as follows:

$$
C_{22}=C_{33}, C_{12}=C_{13}, C_{55}=C_{66}, C_{44}=\left(C_{22}-C_{23}\right) / 2
$$

The stiffness matrix corresponds to a transversely isotropic material with five independent constants. The constitutive equation can be written in matrix form as

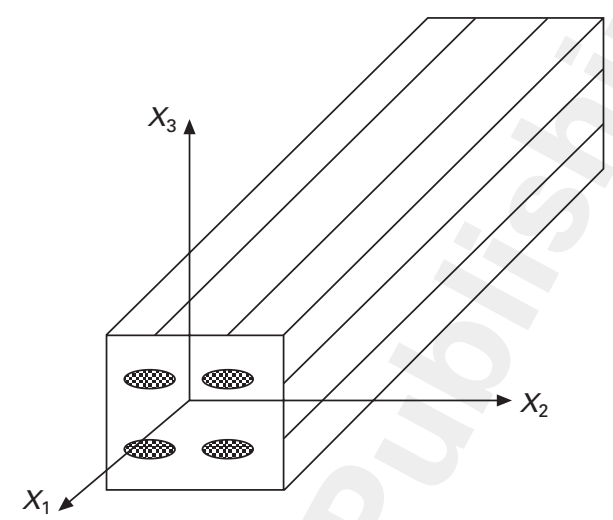

11.7 Example of an orthotropic composite (adapted from [14]).

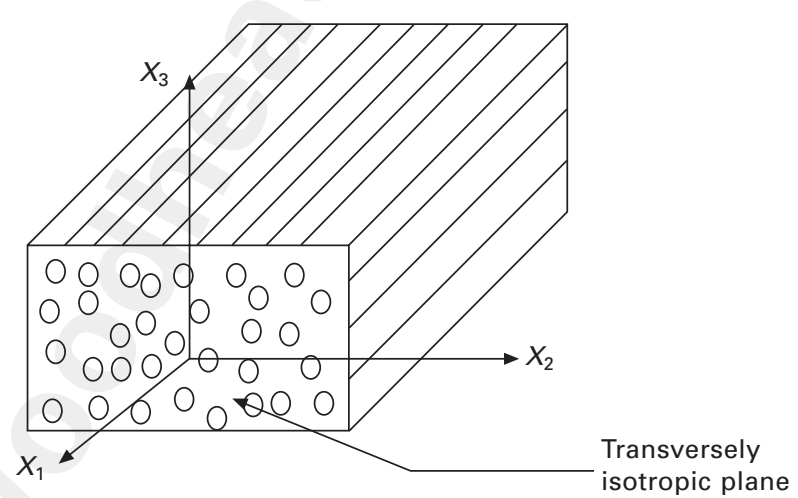

11.8 Transversely isotropic composite (adapted from [14]).

(C) Woodhead Publishing Limited, 2013 


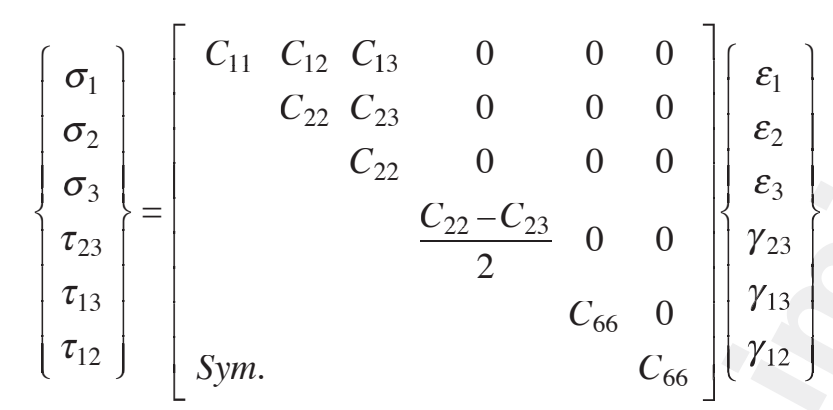

The compliance matrix for a transversely isotropic material in terms of Engineering Constants is given as

$$
\mathbf{S}=\left[\begin{array}{rrrrr}
\frac{1}{E_{1}} & -\frac{v_{12}}{E_{2}}-\frac{v_{12}}{E_{1}} & 0 & 0 & 0 \\
& \frac{1}{E_{2}}-\frac{v_{23}}{E_{2}} & 0 & 0 & 0 \\
& & \frac{1}{E_{2}} & 0 & 0 \\
& & \frac{2\left(1+v_{23}\right)}{E_{2}} & 0 & 0 \\
& & & \frac{1}{G_{12}} & 0 \\
\text { Sym. } & & & & \frac{1}{G_{12}}
\end{array}\right]
$$

The correspondent stiffness matrix is given as

$$
\mathbf{C}=\left[\begin{array}{cccccc}
\frac{1-v_{23}}{\Delta_{1}} E_{1} & \frac{v_{12}}{\Delta_{1}} E_{2} & \frac{v_{12}}{\Delta_{1}} E_{2} & 0 & 0 & 0 \\
\frac{E_{1}-v_{12}^{2} E_{2}}{\Delta_{2}} \frac{E_{1} v_{23}+v_{12}^{2} E_{2}}{\Delta_{2}} & 0 & 0 & 0 \\
& \frac{E_{1}-v_{12}^{2} E_{2}}{\Delta_{2}} & 0 & 0 & 0 \\
& & \frac{1}{2} \frac{E_{2}}{1+v_{23}} & 0 & 0 \\
& & & G_{12} & 0 \\
& & & & & \\
& & & & & \\
& & & & & \\
& & & &
\end{array}\right.
$$


where

$$
\Delta_{1}=\frac{E_{1}\left(1-v_{23}\right)-2 v_{12}^{2} E_{2}}{E_{1}}, \Delta_{2}=\frac{E_{1}\left(1-v_{23}^{2}\right)-2 v_{12}^{2}\left(1+v_{23}\right) E_{2}}{E_{2}}
$$

If all planes are planes of isotropy then the material is called isotropic. The respective stiffness matrix has two independent constants. The additional simplifications on elastic constants are obtained as follows:

$$
C_{11}=C_{22}, C_{12}=C_{23}, C_{66}=\left(C_{22}-C_{23}\right) / 2=\left(C_{11}-C_{12}\right) / 2
$$

The constitutive equation can be written in matrix form as

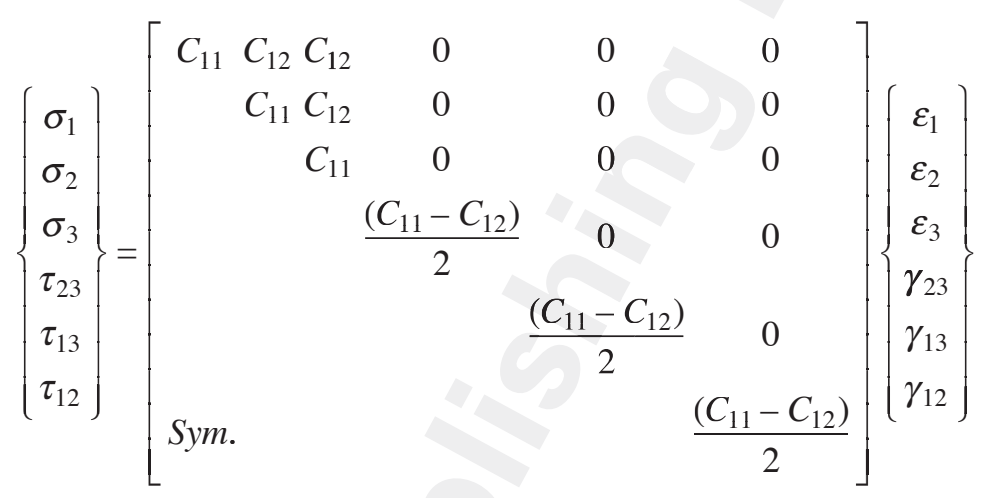

The compliance matrix for an isotropic material in terms of Engineering Constants is given as

$$
\mathbf{S}=\left[\begin{array}{cccccc}
\frac{1}{E} & -\frac{v}{E} & -\frac{v}{E} & 0 & 0 & 0 \\
& \frac{1}{E} & -\frac{v}{E} & 0 & 0 & 0 \\
& \frac{1}{E} & 0 & 0 & 0 \\
& & \frac{2(1+v)}{E} & 0 & 0 \\
& & & \frac{2(1+v)}{E} & 0 \\
\text { Sym. } & & & & \frac{2(1+v)}{E}
\end{array}\right]
$$

The correspondent stiffness matrix is given as 


$$
\mathbf{C}=\left[\begin{array}{rrrrr}
\frac{(1-v) E}{(1-2 v)(1+v)} \frac{v E}{(1-2 v)(1+v)} \frac{v E}{(1-2 v)(1+v)} & 0 & 0 & 0 \\
\frac{(1-v) E}{(1-2 v)(1+v)} \frac{v E}{(1-2 v)(1+v)} & 0 & 0 & 0 \\
\frac{(1-v) E}{(1-2 v)(1+v)} & 0 & 0 & 0 \\
& \frac{E}{2(1+v)} & 0 & 0 \\
& & \frac{E}{2(1+v)} & 0 \\
& & & \frac{E}{2(1+v)}
\end{array}\right]
$$

The relationships between elastic constants which must be satisfied for an isotropic material impose restrictions on the possible range of values for the Poisson's ratio of $-1<v<\frac{1}{2}$. In a similar manner, there are restrictions in orthotropic and transversely isotropic materials. These constraints are based on considerations of the first law of thermodynamics [15]. Moreover, these constraints imply that both the stiffness and compliance matrices must be positive-definite, i.e. each major diagonal term of both matrices must be greater than 0 .

\subsection{Micromechanical analysis of a lamina}

The engineering properties of interest are the elastic constants in the principal material coordinates. If we restrict ourselves to transversely isotropic materials, the elastic properties needed are $E_{1}, E_{2}, v_{12}, G_{12}$ and $G_{23}$, i.e. the axial modulus, the transverse modulus, the major Poisson's ratio, the in-plane shear modulus and the transverse shear modulus, respectively. All the elastic properties can be obtained from these five elastic constants. Since experimental evaluation of these parameters is costly and time-consuming, it becomes important to have analytical models to compute these parameters based on the elastic constants of the individual constituents of the composite. The goal of micromechanics here is to find the elastic constants of the composite as functions of the elastic constants of its constituents, as

$$
C_{i j}^{*}=C_{i j}^{*}\left(E_{1}^{f}, E_{2}^{f}, v_{12}^{f}, v_{23}^{f}, G_{12}^{f}, V_{f}, E^{m}, v^{m,} V_{m}\right)
$$

assuming that the fibres are transversely isotropic and the matrix isotropic. In practice, glass fibres are isotropic and carbon fibres are transversely isotropic. 
The superscripts $f$ and $m$ over the elastic constants stand for fibre and matrix, respectively. The theoretical formulas to compute the elastic properties of a lamina are also dependent on fibre and matrix volume fractions. The fibre volume fraction and the matrix volume fraction are noted as $V_{f}$ and $V_{m}$, respectively. The sum of volume fractions is

$$
V_{f}+V_{m}=1
$$

As a consequence of the manufacturing process of a composite, voids are created in the composite. This causes the theoretical volume of the composite to be lower than the actual volume. Moreover, the void content of a composite decreases the matrix-dominated strengths and the compression strength.

Simple models based on the mechanics of materials approach, semiempirical approaches, and other methods based on advanced topics such as elasticity, will be presented in this section, which is focused mainly on a unidirectional continuous fibre-reinforced lamina. This forms the basic building block of a composite structure which in general is composed of several unidirectional laminae stacked at different angles. A unidirectional lamina is not homogeneous, but it can be assumed homogeneous if the average response of the lamina to mechanical loads is of interest. In this case the elastic properties are assumed to be constant along the lamina, i.e. not depending on the specific location in the lamina.

\subsubsection{Strength of materials approximations}

Approximate formulae for four $\left(E_{1}^{*}, E_{2}^{*}, v_{12}^{*}, G_{12}^{*}\right)$ of the five elastic properties of a transversely isotropic composite can be developed using simple approaches based on the strength of materials concepts. These concepts do not necessarily satisfy in full all the elasticity requirements. The RVE considered consists of a uniform arrangement of straight, continuous fibres.

\section{Effective axial modulus, $E_{l}^{*}$}

Let us assume a transversely unidirectional lamina under uniform axial loading $\bar{\varepsilon}_{1}$, as depicted in Figure 11.9. Assuming that the fibres and matrix are perfectly bonded with no slip, i.e. the axial strain of the composite is uniform,

$$
\bar{\varepsilon}_{1}=\frac{\delta H_{m}}{H}=\frac{\delta H_{f}}{H}
$$

or

$$
\bar{\varepsilon}_{1}=\varepsilon_{m=} \varepsilon_{f}
$$

The axial force equilibrium condition requires that the average axial composite stress $\bar{\sigma}_{1}$ be given as 


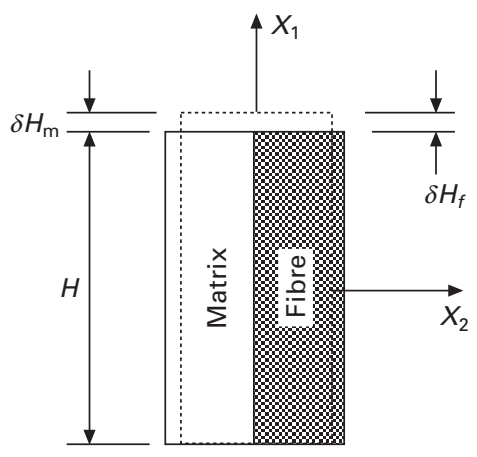

11.9 Axial displacements (adapted from [14]).

$$
\bar{\sigma}_{1} A=\sigma_{m} A_{m} \sigma_{f} A f
$$

with $A=A_{m}+A_{f}$ where, $A, A_{m}$ and $A_{f}$ represent the composite area, matrix area and fibre area, respectively. These are naturally related to the respective volumes. Invoking Hooke's law, the composite axial modulus is given as

$$
\begin{aligned}
E_{1}^{*} & =\frac{\bar{\sigma}_{1}}{\bar{\varepsilon}_{1}}=\frac{\sigma_{m}}{\varepsilon_{m}} \frac{A_{m}}{A}+\frac{\sigma_{f}}{\varepsilon_{f}} \frac{A_{f}}{A}=E^{m} V_{m}+E_{1}^{f} V_{f} \\
& =E^{m}\left(1-V_{f}\right)+E_{1}^{f} V_{f}
\end{aligned}
$$

This result is often designated the rule of mixtures. The effective axial modulus is a linear function of the fibre volume fraction.

\section{Effective axial Poisson's ratio, $v_{12}^{*}$}

The effective axial Poisson's ratio is defined as the negative ratio of lateral strain $\bar{\varepsilon}_{2}$ when under axial strain $\bar{\varepsilon}_{1}$ and $\bar{\sigma}_{1} \neq 0$ and all the other stresses are identically zero,

$$
v_{12}^{*}=-\frac{\bar{\varepsilon}_{2}}{\bar{\varepsilon}_{1}}
$$

Based on the RVE depicted in Fig. 11.10, the following is obtained:

$$
\bar{\varepsilon}_{2}=\frac{\delta H}{H}=\frac{\delta H_{f}+\delta H_{m}}{H}=\frac{-\left(v_{f} \bar{\varepsilon}_{1} V_{f} H+v_{m} \bar{\varepsilon}_{1} V_{m} H\right)}{H}
$$

Therefore the effective axial Poisson's ratio is given as

$$
v_{12}^{*}=v^{m} V_{m}+v_{12}^{f} V_{f}=v^{m}\left(1-V_{f}\right)+v_{12}^{f} V_{f}
$$

As before, this result is often designated the rule of mixtures. The effective axial Poisson's ratio is a linear function of the fibre volume fraction. 


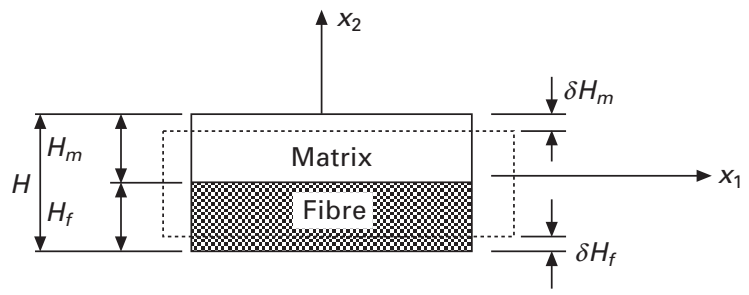

11.10 Transverse displacements (adapted from [14]).

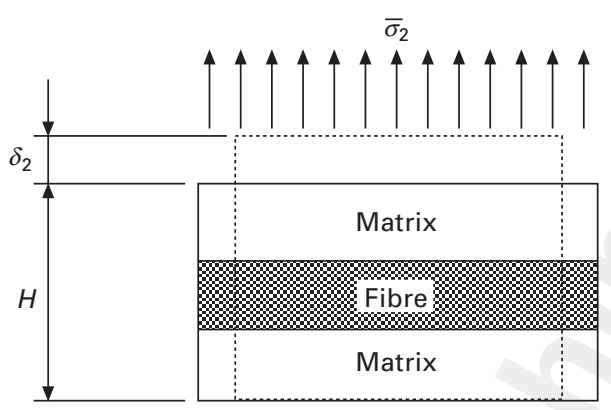

11.11 Transverse loading (adapted from [14]).

Effective transverse modulus, $E_{2}^{*}$

The effective transverse modulus can be determined assuming that the composite is subjected to a uniform transverse stress $\bar{\sigma}_{2}$, as depicted in Fig. 11.11. In this case the transverse stress is constant across the composite; therefore the total transverse deformation for a thickness $H$ is given as

$$
\delta_{2}=\bar{\varepsilon}_{2} H=\varepsilon_{f} V_{f} H+\varepsilon_{m} V_{m} H
$$

Therefore

$$
\bar{\varepsilon}_{2}=\varepsilon_{f} V_{f}+\varepsilon_{m} V_{m}
$$

Consequently the effective transverse modulus is given as

$$
\bar{\varepsilon}_{2}=\frac{\bar{\sigma}_{2}}{E_{2}^{*}}=\frac{\bar{\sigma}_{2}}{E_{2}^{f}} V_{f}+\frac{\bar{\sigma}_{2}}{E_{m}} V_{m}
$$

and

$$
\frac{1}{E_{2}^{*}}=\frac{V_{f}}{E_{2}^{f}}+\frac{V_{m}}{E^{m}}
$$

or

$$
E_{2}^{*}=\frac{E^{m}}{V_{f}\left(\frac{E^{m}}{E_{2}^{f}}-1\right)+1}
$$


The effective transverse modulus is a nonlinear function of the fibre volume fraction.

Effective in-plane shear modulus, $G_{12}^{*}$

The effective in-plane shear modulus can be determined assuming that the composite is subjected to a uniform shear stress $\bar{\tau}_{12}$, as depicted in Fig. 11.12. In this case the shear stress is uniform across the composite; the shear strains in the fibre and matrix are

$$
\gamma_{m}=\frac{\bar{\tau}_{12}}{G^{m}}, \gamma_{f}=\frac{\bar{\tau}_{12}}{G_{12}^{f}}
$$

The average composite shear strain is given by

$$
\tan \bar{\gamma}_{12} \cong \bar{\gamma}_{12}=\frac{\bar{\delta}}{H}
$$

From Fig. 11.12 the following is obtained:

$$
\bar{\delta}=\delta_{f}+\delta_{m}=\frac{\bar{\tau}_{12}}{G_{12}^{f}} V_{f} H+\frac{\bar{\tau}_{12}}{G^{m}} V_{m} H
$$

Therefore

$$
\frac{1}{G_{12}^{*}}=\frac{\bar{\tau}_{12}}{\bar{\gamma}_{12}}=\frac{V_{f}}{G_{12}^{f}}+\frac{V_{m}}{G^{m}}
$$

or

$$
G_{12}^{*}=\frac{G^{m}}{V_{f}\left(\frac{G^{m}}{G_{12}^{f}}-1\right)+1}
$$

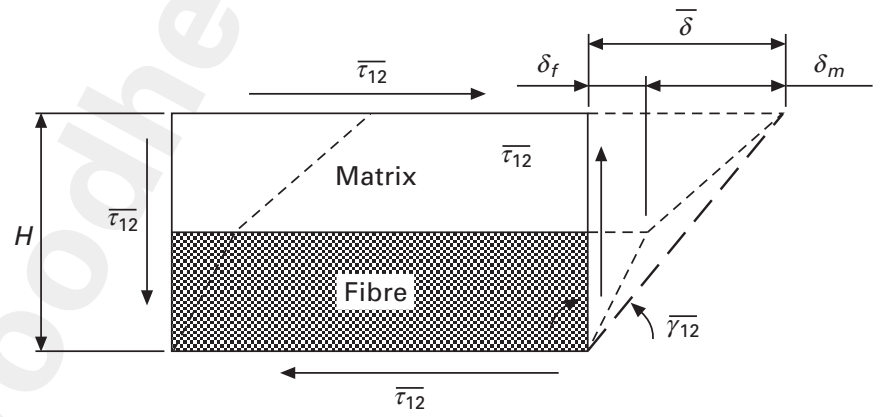

11.12 Shear deformations (adapted from [14]).

(c) Woodhead Publishing Limited, 2013 
As before, the effective in-plane shear modulus is a nonlinear function of the fibre volume fraction.

\subsubsection{Improvements to the strength of materials approximations}

The strength of materials approach provides four of the five elastic properties of transversely isotropic unidirectional composites. Two properties $\left(E_{1}^{*}, v_{12}^{*}\right)$ are well predicted by this simple approach, i.e. using the law of mixtures. The other two $\left(E_{2}^{*}, G_{12}^{*}\right)$ require more accurate micromechanics models. The main reason for this is that $E_{1}^{*}$ and $v_{12}^{*}$ are independent of fibre packing while $\mathrm{d} E_{2}^{*}$ and $G_{12}^{*}$ epend $P$ ngly on fibre arrangement.

The maximum volume fraction of fibres to incorporate in a composite has both theoretical and practical limitations. The possible fibre arrangements lead to these limitations. Some examples of fibre packing geometry are shown in Fig. 11.13.

A theoretical maximum fibre volume fraction can be calculated for each packing geometry. For the square array and close packing shown in Fig. 11.14 , the maximum fibre volume fraction is given as

$$
\begin{aligned}
& \text { Square array: }\left(V_{f}\right)_{\max }=\frac{\pi D^{2} / 4}{D^{2}}=0.785 \\
& \text { Close packing: }\left(V_{f}\right)_{\max }=\frac{\pi D^{2} / 4}{\sqrt{3} D^{2} / 4}=0.907
\end{aligned}
$$

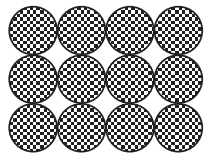

Square array

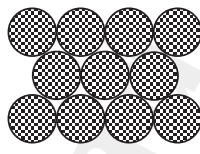

Close packing

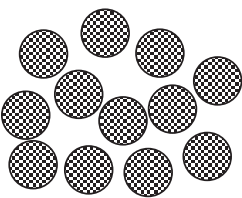

Random

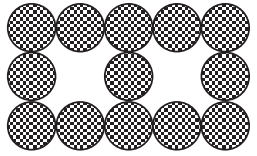

Regular open

11.13 Examples of fibre packing.

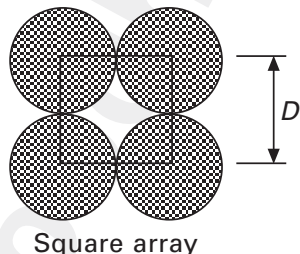

Square array

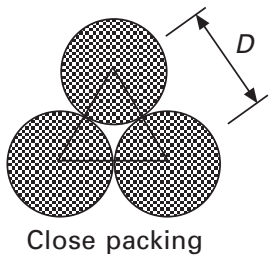

Close packing

11.14 Geometry of fibre packing for two cases: square array and close packing. 
The approach employed to improve the formulas for the two elastic properties is based on the square array. Referring to the RVE represented in Fig. 11.15, since the theoretical fibre volume fraction is given by Equation (11.42) we must have $s=d$.

Now it is possible to proceed to divide the RVE into two regions [15], according to Fig. 11.16. The square dimension of the fibre becomes

$$
s_{f}=\sqrt{\frac{\pi}{4}} d
$$

Therefore the RVE dimension becomes

$$
s_{f}=\sqrt{\frac{\pi}{4 V_{f}}} d
$$

Noting that

$$
\frac{s_{f}}{s}=\sqrt{V_{f}}
$$

then

$$
\frac{s_{m}}{s}=1-\sqrt{V_{f}}
$$

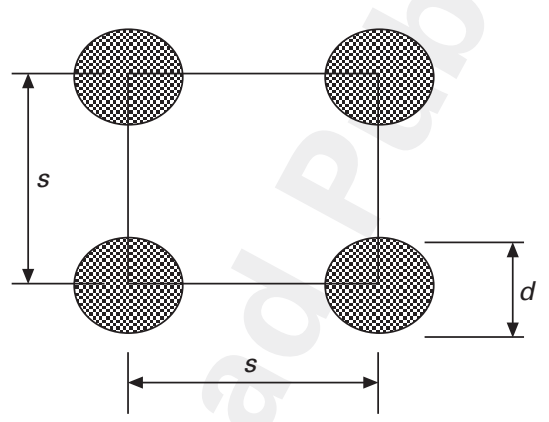

11.15 Representative volume element used for square array.
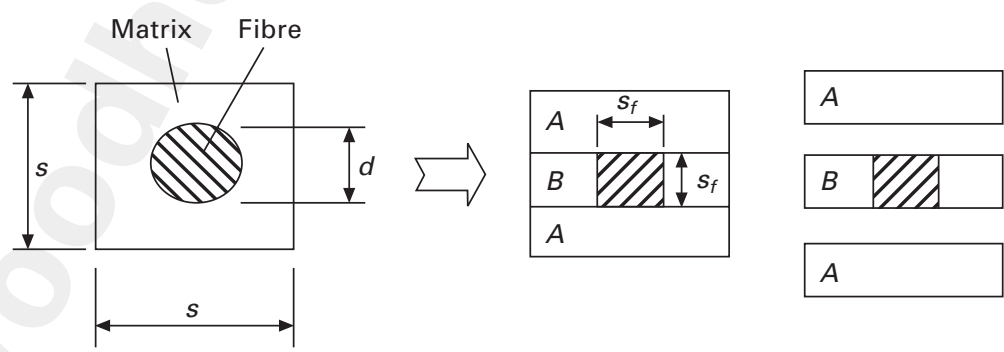

$A$

11.16 Division of RVE into two regions ( $A$ and $B)$.

(C) Woodhead Publishing Limited, 2013 
In region $B$ the effective transverse modulus is obtained by strength of materials analysis:

$$
\frac{1}{E_{B 2}}=\frac{s_{f} / s}{E_{2}^{f}}+\frac{s_{m} / s}{E^{m}}=\frac{1-\sqrt{V_{f}}\left(1-E^{m} / E_{2}^{f}\right)}{E^{m}}
$$

A parallel combination of regions $A$ and $B$ is under a transverse normal stress, where the rule of mixtures can be applied to allow the determination of the effective transverse modulus:

$$
E_{2}^{*}=E_{B 2} \frac{s_{f}}{s}+E^{m} \frac{s_{m}}{s}=\frac{E^{m}}{1-\sqrt{V_{f}}\left(1-E^{m} / E_{2}^{f}\right)} \sqrt{V_{f}}+E^{m}\left(1-\sqrt{V_{f}}\right)
$$

and finally

$$
E_{2}^{*}=E^{m}\left[\frac{\sqrt{V_{f}}}{\sqrt{V_{f}} E^{m} / E_{2}^{f}+\left(1-\sqrt{V_{f}}\right)}+\left(1-\sqrt{V_{f}}\right)\right]
$$

For $G_{12}^{*}$ a similar expression can be found. These results are close to the Chamis formulae [16],

$$
E_{2}^{*}=\frac{E^{m}}{\sqrt{V_{f}} E^{m} / E_{2}^{f}+\left(1-\sqrt{V_{f}}\right)}
$$

\subsubsection{Spencer's approach}

Another approach to improve the accuracy of the formulae for $E_{2}^{*}$ and $G_{12}^{*}$ was suggested by Spencer [17]. The proposed model was also based on a square array but included the stress concentration effects at the points where the fibres were closer. The formulae are given as

$$
E_{2}^{*}=E^{m}\left[\frac{\Gamma-1}{\Gamma}+\frac{1}{k_{E}}\left(\frac{2 \Gamma}{\sqrt{\Gamma^{2}-k_{E}^{2}}} \arctan \frac{\sqrt{\Gamma+k_{E}}}{\sqrt{\Gamma-k_{E}}}-\frac{\pi}{2}\right)\right]
$$

and

$$
G_{12}^{*}=G^{m}\left[\frac{\Gamma-1}{\Gamma}+\frac{1}{k_{G}}\left(\frac{2 \Gamma}{\sqrt{\Gamma^{2}-k_{G}^{2}}} \arctan \frac{\sqrt{\Gamma+k_{G}}}{\sqrt{\Gamma-k_{G}}}-\frac{\pi}{2}\right)\right]
$$

where

$$
k_{E}=1-\frac{E^{m}}{E_{2}^{f}}, k_{G}=1-\frac{G^{m}}{G_{12}^{f}}, \Gamma=\frac{s}{d}=1
$$


Spencer [17] also suggested that $\Gamma$ can be given for a large variety of fibre packing geometries and respective volume fractions $V_{f}$ by

$$
\Gamma=\frac{1}{\sqrt{V_{f}\left(1.1 V_{f}^{2}-2.1 V_{f}+2.2\right)}}
$$

The evolution of $\Gamma$ is plotted in Fig. 11.17. The deviations from unity become significant for lower fibre volume fractions.

\subsubsection{Continuous approaches}

In a critical review by Chamis and Sendeckyj [18], it was proposed to classify the variety of micromechanical approaches as follows: netting analysis, mechanics of materials (or strength of materials), self-consistent model, variational, elasticity, statistical, discrete element and semi-empirical methods, and theories accounting for microstructure. After this Mori and Tanaka [19, 20] proposed an original method which assumes that the average strain in the fibre is related to the average strain in the matrix by a fourth-order tensor. Another approach, designated the method of cells (MOC), was initiated by Aboudi [21] and later extended as the generalized method of cells (GMC) [22]. More recently, a new micromechanics model was developed to overcome the shortcomings of GMC. This model, based on a higher-order theory, is designated the high fidelity generalized method of cells (HFGMC) [23]. All these methods, except netting analysis and mechanics of materials, use some or all of the principles of elasticity to different extents.

\subsubsection{Elasticity model}

The arrangement of fibres in the matrix influences the transverse elastic properties. In a real case this cannot be known precisely and various

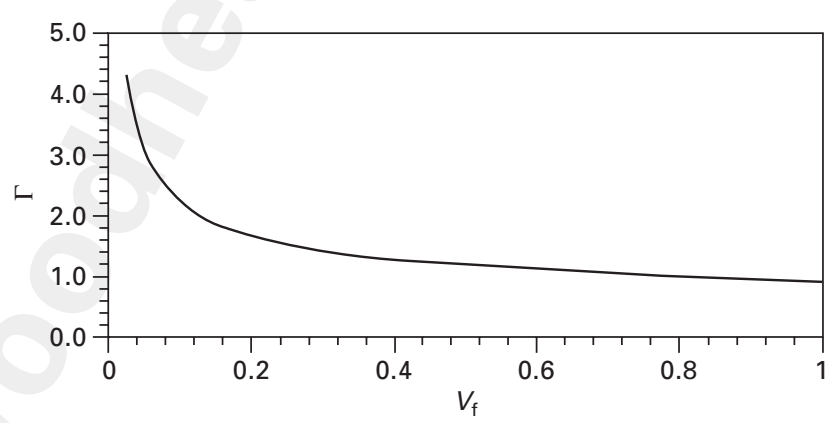

11.17 Dependence of $\Gamma$ on fibre content.

(C) Woodhead Publishing Limited, 2013 


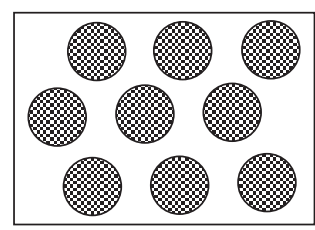

$C=0$

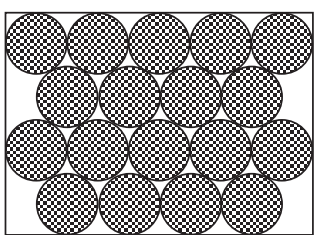

$C=1$

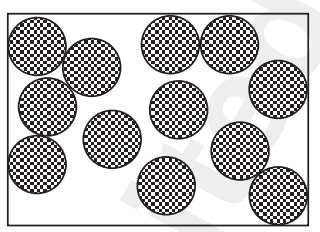

$0<C<1$

11.18 Contiguity models for calculating transverse elastic properties.

approaches can be used. Tsai [24] used models of fibre contiguity as depicted in Fig. 11.18.

Two extreme cases can be recognized from Fig. 11.18: when fibres have the maximum number of neighbours touching them $(C=1)$ and when fibres are isolated in the matrix $(C=0)$. Most composites have partial contiguity, therefore $0<C<1$.

The transverse Young's modulus based on an elasticity solution using the contiguity models is given as

$$
\begin{aligned}
& E_{2}^{*}=2\left[1-v_{12}^{f}\right. \\
& \left.+\left(v_{12}^{f}-v^{m}\right) V_{m}\right] E^{m}\left[\begin{array}{c}
(1-C) \frac{K_{f}\left(2 K_{m}+G^{m}\right)-G_{m}\left(K_{f}-K_{m}\right) V_{m}}{\left(2 K_{m}+G^{m}\right)+2\left(K_{f}-K_{m}\right) V_{m}} \\
+C \frac{K_{f}\left(2 K_{m}+G_{12}^{f}\right)+G_{12}^{f}\left(K_{m}-K_{f}\right) V_{m}}{\left(2 K_{m}+G_{12}^{f}\right)-2\left(K_{m}-K_{f}\right) V_{m}}
\end{array}\right]
\end{aligned}
$$

where

$$
K_{f}=\frac{E_{2}^{f}}{2\left(1-v_{12}^{f}\right)}, K_{m}=\frac{E^{m}}{2\left(1-v^{m}\right)}, G_{12}^{f}=\frac{E_{2}^{f}}{2\left(1+v_{12}^{f}\right)}, G^{m}=\frac{E^{m}}{2\left(1+v^{m}\right)}
$$

In this solution the fibres are assumed isotropic, i.e. $E_{1}^{f}=E_{2}^{f}=E_{3}^{f}$ and $v_{12}^{f}$ $=v_{23}^{f}$.

The effective planar shear modulus is

$$
\begin{aligned}
G_{12}^{*}= & G^{m} \frac{2 G_{12}^{f}-\left(G_{12}^{f}-G^{m}\right) V_{m}}{2 G^{m}+\left(G_{12}^{f}-G^{m}\right) V_{m}} \\
& (1-C)+G_{12}^{f} \frac{G^{m}+G_{12}^{f}-\left(G_{12}^{f}-G^{m}\right) V_{m n}}{G^{m}+G_{12}^{f}+\left(G_{12}^{f}-G^{m}\right) V_{m}} C
\end{aligned}
$$

The Poisson's ratio is 


$$
\begin{aligned}
v_{12}^{*}= & (1-C) \frac{K_{f} v_{12}^{f}\left(2 K_{m}+G^{m}\right) V_{f}+K_{m} v^{m}\left(2 K_{f}+G^{m}\right) V_{m}}{K_{f}\left(2 K_{m}+G^{m}\right)-G^{m}\left(K_{f}-K_{m}\right) V_{m}} \\
& +C \frac{K_{m} v^{m}\left(2 K_{f}+G_{12}^{f}\right) V_{m}+K_{f} v_{12}^{f}\left(2 K_{m}+G_{12}^{f}\right) V_{f}}{K_{f}\left(2 K_{m}+G_{12}^{f}\right)+G_{12}^{f}\left(K_{m}-K_{f}\right) V_{m}}
\end{aligned}
$$

\subsubsection{Halpin-Tsai equations}

Due to the inherent complexity of previous developments and the uncertainty associated with the contiguity parameter, a simplified approach was obtained from the elasticity. The elastic constants $E_{1}^{*}$ and $v_{12}^{*}$ are given by the law of mixtures which proved to be accurate enough. The remaining elastic constants, $E_{2}^{*}, G_{12}^{*}$ and $v_{23}^{*}$, are given by

$$
\begin{aligned}
& E_{2}^{*}=E^{m} \frac{1+\xi \eta V_{f}}{1-\eta V_{f}} \text { with } \eta=\frac{E_{2}^{f} / E^{m}-1}{E_{2}^{f} / E^{m}-} \\
& G_{12}^{*}=G^{m} \frac{1+\xi \eta V_{f}}{1-\eta V_{f}} \text { with } \eta=\frac{G_{12}^{f} / G^{m}-1}{G_{12}^{f} / G^{m}-} \\
& v_{12}^{*}=v^{m} \frac{1+\xi \eta V_{f}}{1-\eta V_{f}} \text { with } \eta=\frac{v_{23}^{f} / \nu^{m}-1}{v_{23}^{f} / \nu^{m}+\xi}
\end{aligned}
$$

The parameter $\xi$ is a measure of fibre reinforcement of the composite materials that depends on several factors, such as fibre geometry and fibre arrangement. The exact elasticity calculations predict the moduli to rise faster with increasing fibre volume fraction above 0.7 as compared to the Halpin-Tsai equation [25]. The following empirical expressions were suggested by others [26]:

$$
\xi_{E_{2}}=1+40 V_{f}^{10}, \xi_{G_{12}}=2+40 V_{f}^{10}
$$

\subsubsection{Additional approaches}

As discussed before, the rule of mixtures proved to give accurate predictions for the longitudinal modulus and Poisson's ratio. The plane shear modulus can be predicted with good accuracy by the self-consistent model [10, 27]:

$$
G_{12}^{*}=G^{m}\left[\frac{G^{m}\left(1-V_{f}\right)+G_{12}^{f}\left(1+V_{f}\right)}{G^{m}\left(1+V_{f}\right)+G_{12}^{f}\left(1-V_{f}\right)}\right]
$$

For the other elastic properties, the self-consistent model is only able to give accurate predictions for the plane-strain bulk modulus, 


$$
k_{23}^{*}=\frac{k^{m}\left(k_{23}^{f}+G^{m}\right)\left(1-V_{f}\right)+k_{23}^{f}\left(k^{m}+G^{m}\right) V_{f}}{\left(k_{23}^{f}+G^{m}\right)\left(1-V_{f}\right)+\left(k^{m}+G^{m}\right) V_{f}}
$$

The plane-strain bulk modulus is related to the other elastic properties by [14]:

$$
k_{23}^{*}=\frac{1}{2}\left(C_{22}^{*}+C_{23}^{*}\right)=\frac{E_{1}^{*} E_{2}^{*}}{2\left(E_{1}^{*}-E_{1}^{*} v_{23}^{*}-2 E_{2}^{*}\left(v_{12}^{*}\right)^{2}\right)}
$$

In a similar fashion the plane bulk modulus of the matrix (isotropic) and fibre (transversely isotropic) are given by

$$
k^{m}=\frac{E^{m}}{2\left(1+v^{m}\right)\left(1-2 v^{m}\right)}, k_{23}^{f}=\frac{E_{1}^{f} E_{2}^{f}}{2\left(E_{1}^{f}-E_{1}^{f} v_{23}^{f}-2 E_{2}^{f}\left(v_{12}^{f}\right)^{2}\right)}
$$

In this context Morais [27] derived a closed-form equation for the transverse modulus using a relatively simple mechanics-of-materials model. The method employed is a sophistication of the technique presented in Section 11.4.2. The formula obtained for the transverse modulus is given by

$$
E_{2}^{*}=E^{m}\left[\frac{\sqrt{V_{f}}}{\sqrt{V_{f}} E^{m} / E_{2}^{f}+\left(1-\sqrt{V_{f}}\right)\left(1-\left(v^{m}\right)^{2}\right)}+\frac{\left(1-\sqrt{V_{f}}\right)}{1-\left(v^{m}\right)^{2}}\right][
$$

From these results it becomes straightforward to obtain the transverse Poisson's ratio modulus:

$$
v_{23}^{*}=1-E_{2}^{*}\left(\frac{2\left(v_{12}^{*}\right)^{2}}{E_{1}^{*}}+\frac{1}{2 k_{23}^{*}}\right)
$$

and then the transverse shear modulus:

$$
G_{23}^{*}=\frac{E_{2}^{*}}{2(1-2)}
$$

Another model based on ideas similar to those presented in section 11.4.2 was obtained by Fu et al. [28] for the transverse modulus:

$$
\frac{1}{E_{2}^{*}}=\frac{1}{E^{m}}\left[\frac{\sqrt{4 V_{f} / \pi}}{\sqrt{\pi V_{f} / 4} E_{2}^{f} / E^{m}+\left(1-\sqrt{\pi V_{f} / 4}\right)}+\left(1-\sqrt{4 V_{f} / \pi}\right]\right.
$$

Neither formula provides coherent results for the extreme values of fibre volume fraction $V_{f}$, i.e. $\lim _{V_{f} \rightarrow 0} E_{2}^{*}=\frac{E^{m}}{1-\left(v^{m}\right)^{2}} \neq E^{m}$ for Equation (11.64), and $\lim _{V_{f} \rightarrow 1} \frac{1}{E_{2}^{*}}=\frac{1}{E^{m}}\left[\frac{4}{\pi E_{2}^{f} / E^{m}+(2 \sqrt{\pi}-\pi)}+\left(1-\frac{2}{\sqrt{\pi}}\right)\right] \neq \frac{1}{E_{2}^{f}}$ for Equation (11.67). 
In this field there are several other related works. Among them and not so well known is the work of Puck in which models were developed to predict elastic properties of unidirectional fibre-reinforced composites [29]. His work was based on glass fibre-reinforced composites and therefore assumes that both constituents, fibre and matrix, are isotropic materials.

\subsubsection{Bridging model}

A unified micromechanics model named the Bridging Model was developed by Huang [30]. The main feature of the model is to correlate the averaged stress states in the constituent fibre and matrix through a bridging matrix. This matrix depends only on the constituent properties and on the fibre packing geometry in the matrix. The model recovers the rule of mixtures formulae for the axial modulus and the major Poisson's ratio $\left(E_{1}^{*}, v_{12}^{*}\right)$. The formula for the in-plane shear modulus $\left(G_{12}^{*}\right)$ recovers the result obtained by the self-consistent model. The differences arise for the remaining elastic constants - the transverse modulus,

$$
E_{2}^{*}=\frac{\left(V_{f}+\left(1-V_{f}\right) a_{11}\right)\left(V_{f}+\left(1-V_{f}\right) a_{22}\right)}{\left(V_{f}+\left(1-V_{f}\right) a_{11}\right)\left(V_{f} S_{22}^{f}+\left(1-V_{f}\right) a_{22} S_{22}^{m}\right)+V_{f}\left(1-V_{f}\right) a_{12}\left(S_{12}^{m}-S_{12}^{f}\right)}
$$

and the transverse shear modulus,

$$
G_{23}^{*}=\frac{1}{2} \frac{\left(V_{f}+\left(1-V_{f}\right) a_{22}\right.}{V_{f}\left(S_{22}^{f}-S_{23}^{f}\right)+\left(1-V_{f}\right) a_{22}\left(S_{22}^{m}-S_{23}^{m}\right)}
$$

where

$$
\begin{aligned}
& a_{11}=E^{m} / E_{1}^{f}, a_{22}=\frac{1}{2}\left(1+E^{m} / E_{2}^{f}\right), a_{12}=\frac{\left(S_{12}^{f}-S_{12}^{m}\right)}{\left(S_{11}^{f}-S_{11}^{m}\right)}\left(a_{11}-a_{22}\right) \\
& S_{11}^{f}=1 / E_{1}^{f}, S_{22}^{f}=1 / E_{2}^{f}, S_{12}^{f}=-v_{12}^{f} / E_{2}^{f}, S_{23}^{f}=-v_{23}^{f} / E_{2}^{f} \\
& S_{11}^{m}=S_{22}^{m}=1 / E^{m}, S_{12}^{m}=S_{23}^{m}=-v^{m} / E^{m}
\end{aligned}
$$

\subsection{Comparing micromechanical models with experimental data}

Results obtained from the literature are used to assess the prediction capabilities of the micromechanics approaches. In this case a unidirectional composite made using isotropic glass fibres and epoxy matrix with $E^{f}=73.1 \mathrm{GPa}, v^{f}$ $=0.22, E^{m}=3.45 \mathrm{GPa}$ and $v^{m}=0.35[30]$ is used. The predicted transverse 
modulus $E_{2}^{*}$ is shown in Fig. 11.19. The simple formula given by Chamis [16] proves to give accurate predictions. The rule of mixtures (ROM) prediction underestimates the composite transverse modulus.

Experimental data were obtained for the in-plane shear modulus $G_{12}^{*}$ of another unidirectional composite made of isotropic glass fibres and epoxy matrix with $E \Leftrightarrow 0.2 \mathrm{GPa}$ and $G^{m}=1.8 \mathrm{GPa}$ [31]. The predicted in-plane shear modulus $G_{12}$ is shown in Fig. 11.20. Again, in this case, the simple

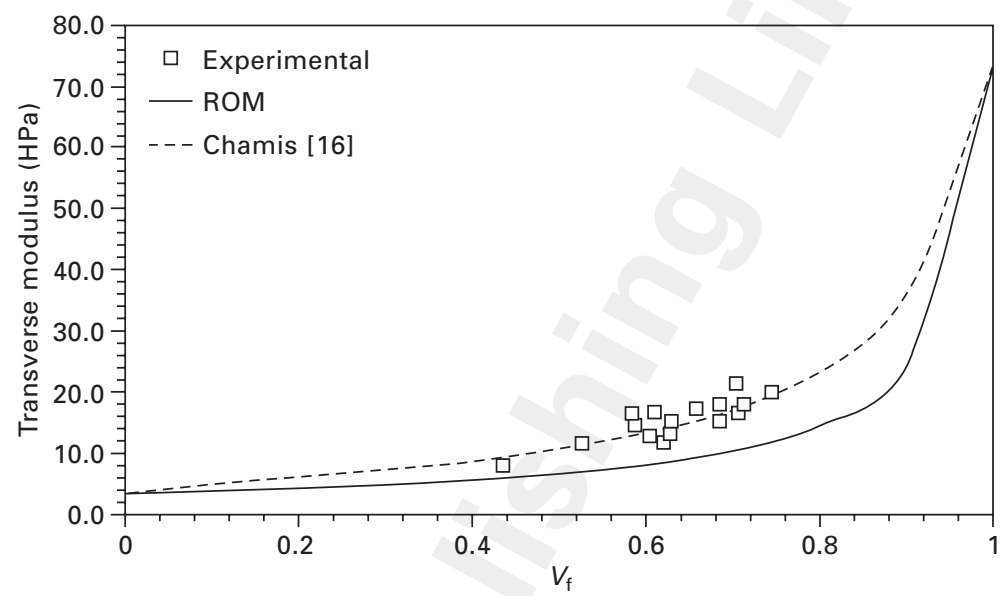

11.19 Transverse modulus $E_{2}^{*}$ of unidirectional glass/epoxy composite as a function of fibre volume fraction $\left(E^{f}=73.1 \mathrm{GPa}, v^{f}=\right.$ $\left.0.22, E^{m}=3.45 \mathrm{GPa}, v^{m}=0.35\right)$.

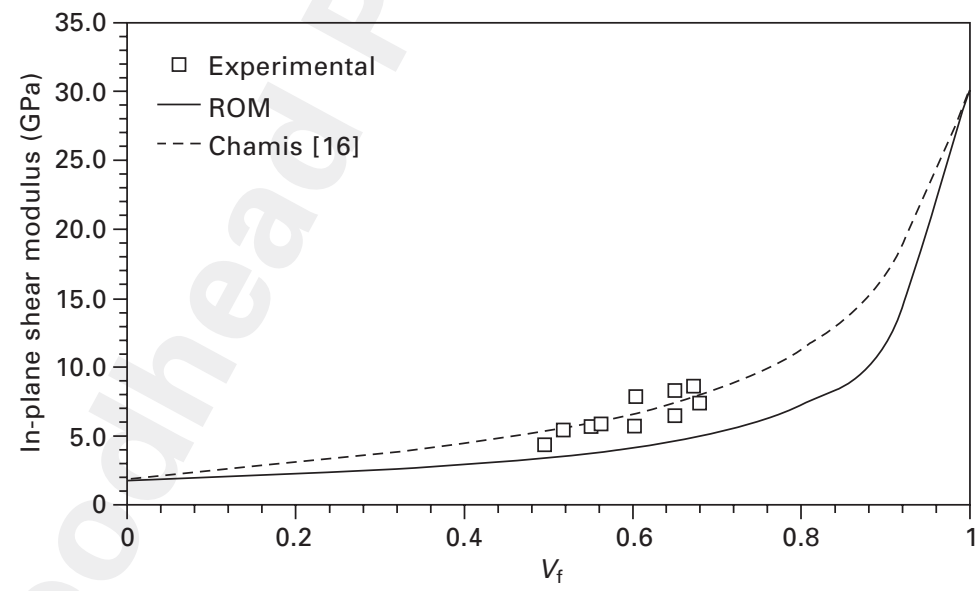

11.20 In-plane shear modulus $G_{12}^{*}$ of unidirectional alass/epoxy composite as a function of fibre volume fraction $E$. $2 \mathrm{GPa}, G^{m}=$ $1.8 \mathrm{GPa})$. 
formula given by Chamis [16] proves to give accurate predictions. Again, the rule of mixtures (ROM) prediction underestimates the composite in-plane shear modulus.

Kriz and Stinchcomb [32] published experimental data for unidirectional graphite/epoxy composites. These results illustrate the case when the fibres are transversely isotropic. The elastic properties of the matrix are $E^{m}=5.28$ $\mathrm{GPa}$ and $v^{m}=0.354$, and for the fibres $E_{1}^{f}=232 \mathrm{GPa}, E_{2}^{f}=15 \mathrm{GPa}, G_{12}^{f}$ $=24 \mathrm{GPa}, v_{12}^{f}=0.279$ and $v_{23}^{f}=0.49$. In Figs $11.21-11.25$ are plotted the predictions against the experimental data for $E_{1}^{*}, E_{2}^{*}, G_{12}^{*}, G_{23}^{*}$ and $v_{23}^{*}$, i.e. the longitudinal or axial modulus, the transverse modulus, the in-plane shear modulus, the transverse shear modulus and the transverse Poisson's ratio, respectively.

For the transverse shear modulus, the approach designated self-consistent was based on the formula obtained by the self-consistent method for the planestrain bulk modulus (11.61), on the transverse modulus calculated using the Chamis approach (11.49b) and the in-plane Poisson's ratio given by the rule of mixtures. Except when used to predict the axial modulus and the major Poisson's ratio, the rule of mixtures underestimates the remaining composite elastic properties. The Bridging Model proved to be a very effective theory to account for all five elastic properties for unidirectional composites that are transversely isotropic.

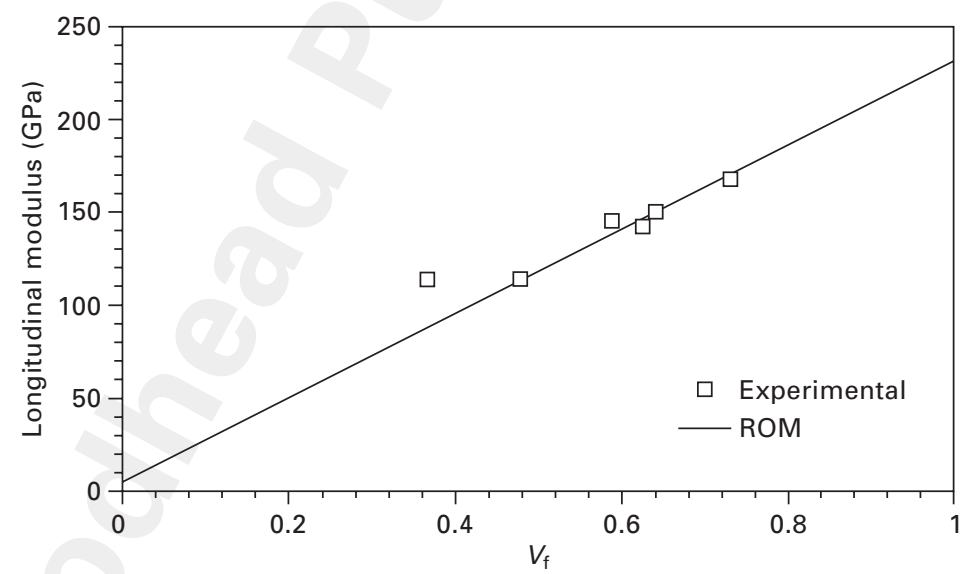

11.21 Longitudinal modulus $E_{1}^{*}$ of unidirectional carbon/epoxy composite as a function of fibre volume fraction $\left(E^{m}=5.28 \mathrm{GPa}, v^{m}\right.$ $=0.354, E_{1}^{f}=232 \mathrm{GPa}, E_{2}^{f}=15 \mathrm{GPa}, G_{12}^{f}=24 \mathrm{GPa}, v_{12}^{f}=0.279$, $\left.v_{23}^{f}=0.49\right)$. 


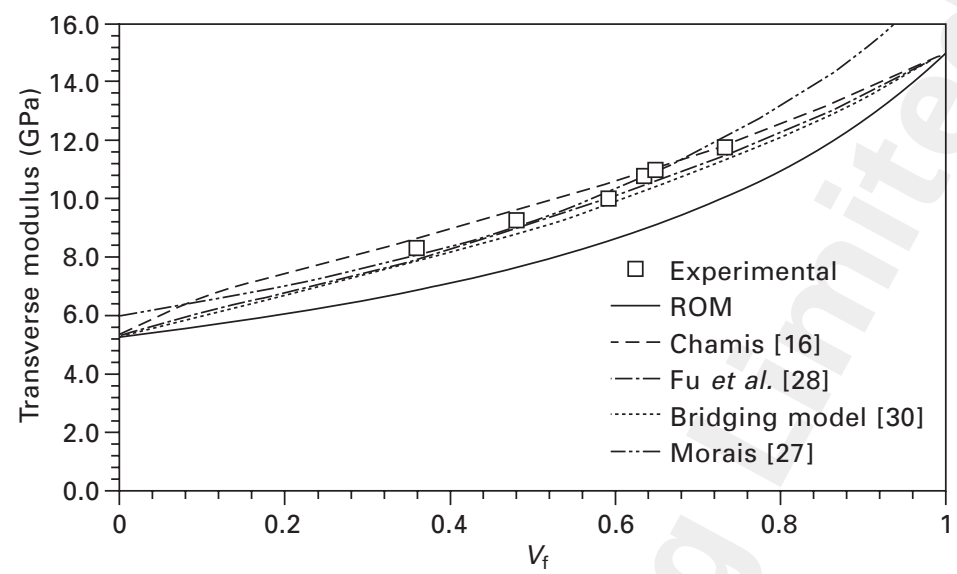

11.22 Transverse modulus $E_{2}^{*}$ of unidirectional carbon/epoxy composite as a function of fibre volume fraction $\left(E^{m}=5.28 \mathrm{GPa}, v^{m}=\right.$ $0.354, E_{1}^{f}=232 \mathrm{GPa}, E_{2}^{f}=15 \mathrm{GPa}, G_{12}^{f} 24 \mathrm{GPa}, v_{12}^{f}=0.279, v_{23}^{f}$ $=0.49$ ).

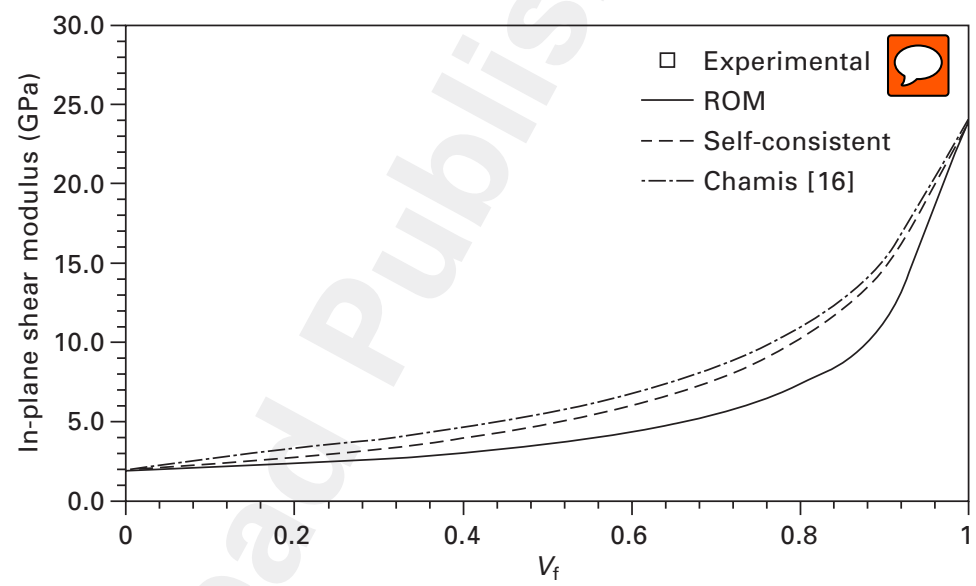

11.23 In-plane shear modulus $G_{12}^{*}$ of unidirectional carbon/epoxy composite as a function of fibre volume fraction $\left(E^{m}=5.28 \mathrm{GPa}, v^{m}\right.$ $=0.354, E_{1}^{f}=232 \mathrm{GPa}, E_{2}^{f}=15 \mathrm{GPa}, G_{12}^{f}=24 \mathrm{GPa}, v_{12}^{f}=0.279$, $\left.v_{23}^{f}=0.49\right)$.

\subsection{Stiffness and compliance transformations}

Stress and strain are second-order tensors while stiffness and compliance are fourth-order tensors [14]. Hence these entities are ruled by the tensor transformation laws that establish the relationships between the components 


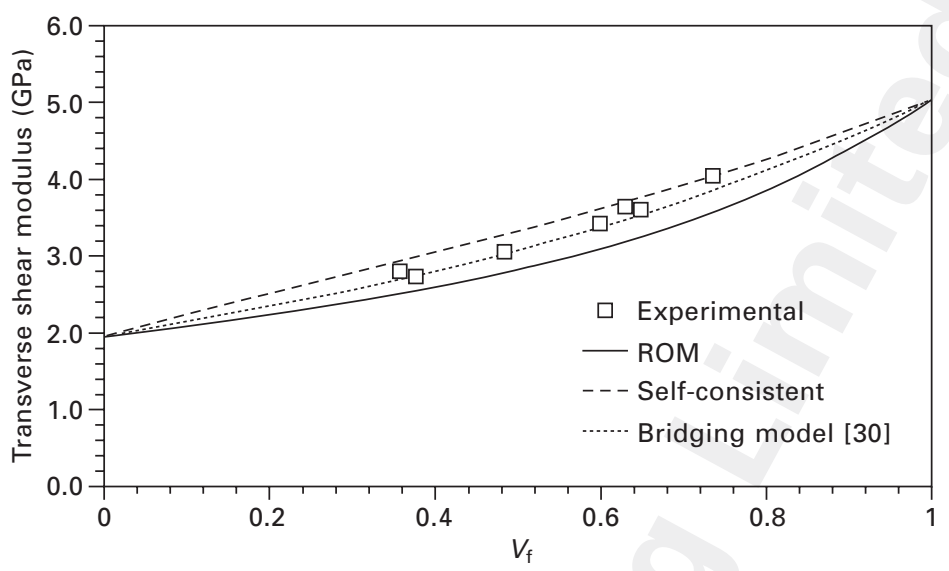

11.24 Transverse shear modulus $G_{23}^{*} \bigcirc$ nidirectional carbon/epoxy composite as a function of fibre volume fraction $\left(E^{m}=5.28 \mathrm{GPa}, v^{m}\right.$ $=0.354, E_{1}^{f}=232 \mathrm{GPa}, E_{2}^{f}=15 \mathrm{GPa}, G_{12}^{f}=24 \mathrm{GPa}, v_{12}^{f}=0.279$, $v_{23}^{f}=0.49$ ).

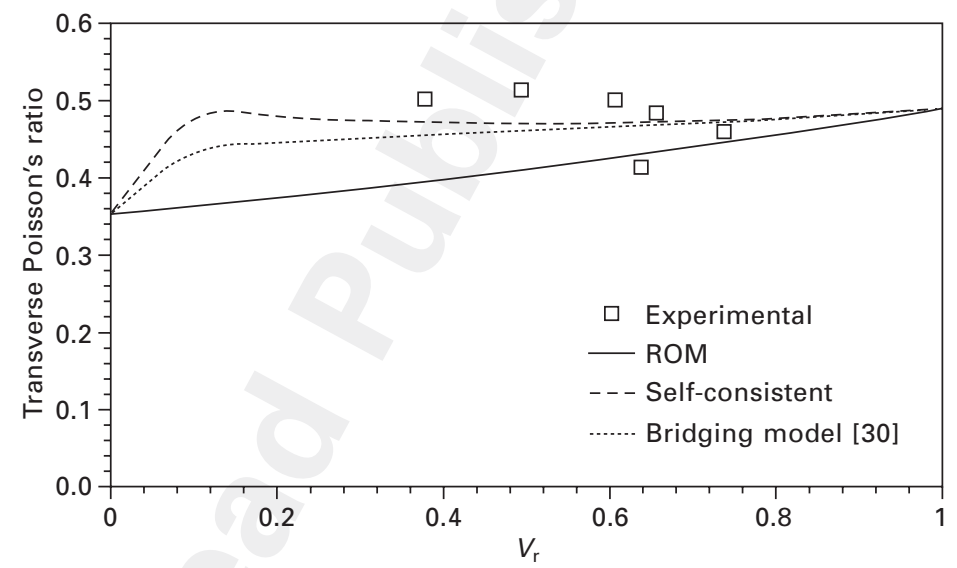

11.25 Transverse Poisson's ratio $v_{23}^{f}$ of unidirectional carbon/epoxy composite as a function of fibre volume fraction $\left(E^{m}=5.28 \mathrm{GPa}, v^{m}\right.$ $=0.354, E_{1}^{f}=232 \mathrm{GPa}, E_{2}^{f}=15 \mathrm{GPa}, G_{12}^{f}=24 \mathrm{GPa}, v_{12}^{f}=0.279$, $\left.v_{23}^{f}=0.49\right)$.

in rotated coordinated systems. The stiffness and compliance tensors are readily obtained from the five independent elastic constants, for the case of transversely isotropic materials. These tensors are obtained for the principal material coordinate system. In the principal material coordinate system, 
formed by the 1-2-3 axes as shown in Fig. 11.26(a), the 1-axis is associated with the maximum lamina stiffness, while the 2-axis corresponds to the direction of minimum lamina stiffness. In laminated composites the laminae are stacked sequentially with different orientations. In this case the material's coordinate system may no longer coincide with the global coordinate system formed by the $x-y-z$ axes as shown in Fig. 11.26(b). This arrangement is designated the off-axis configuration. The angle $\theta$ is measured positive counterclockwise from the $x$-axis to the 1-axis. In the case of Fig. 11.26(b) the angle is negative, i.e. $\theta<0$.

Therefore it becomes necessary to use the transformation laws which relate the tensors in one coordinate system to another in a rotated coordinate system. Briefly, the relationship between the stresses in the principal material and global coordinates [14] is given by

$$
\{\sigma\}_{1}=\left[T_{1}\right]\{\sigma\}_{x}
$$

where the transformation matrix is

$$
\left\lfloor T_{1}\right\rfloor=\left[\begin{array}{cccccc}
m^{2} & n^{2} & 0 & 0 & 0 & 2 m n \\
n^{2} & m^{2} & 0 & 0 & 0 & -2 m n \\
0 & 0 & 1 & 0 & 0 & 0 \\
0 & 0 & 0 & m & -n & 0 \\
0 & 0 & 0 & n & m & 0 \\
-m n & m n & 0 & 0 & 0 & m^{2}-n^{2}
\end{array}\right] \text { with } m=\cos \theta \text { and } n=\sin \theta
$$

The strain transformation is

$$
\{\varepsilon\}_{1}=\left[T_{2}\right]\{\varepsilon\}_{x}
$$

where the transformation matrix is

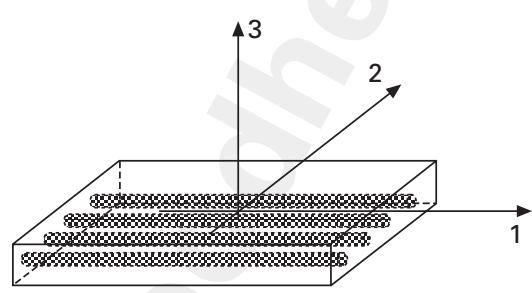

(a)

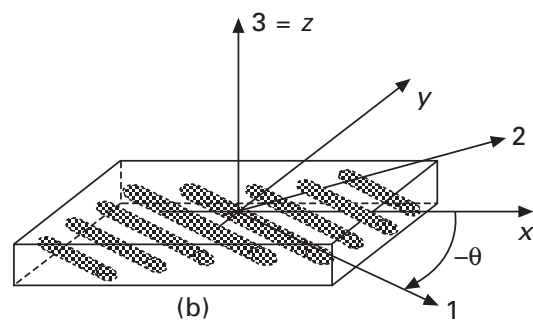

(b)

11.26 Schematic representation of (a) principal material coordinate system (1-2-3) and (b) lamina coordinated system $(x-y-z)$. 


$$
\left\lfloor T_{2}\right\rfloor=\left[\begin{array}{cccccc}
m^{2} & n^{2} & 0 & 0 & 0 & m n \\
n^{2} & m^{2} & 0 & 0 & 0 & -m n \\
0 & 0 & 1 & 0 & 0 & 0 \\
0 & 0 & 0 & m & -n & 0 \\
0 & 0 & 0 & n & m & 0 \\
-2 m n & 2 m n & 0 & 0 & 0 & m^{2}-n^{2}
\end{array}\right]
$$

Therefore the transformed stiffness $[\bar{C}]$ may be determined using the relationships obtained for the stress and strain transformation,

$$
\begin{aligned}
\{\sigma\}_{1} & =[C]\{\varepsilon\}_{1} \Leftrightarrow\left[T_{1}\right]\{\sigma\}_{x}=[C]\left[T_{2}\right]\{\varepsilon\}_{x} \Leftrightarrow\{\sigma\}_{x} \\
& =\left[T_{1}\right]^{-1}[C]\left[T_{2}\right]\{\varepsilon\}_{x}
\end{aligned}
$$

Therefore,

$$
\begin{aligned}
& {[\bar{C}]=\left[T_{1}\right]^{-1}[C]\left[T_{2}\right], \text { noting that }} \\
& {\left[T_{i}(\theta)\right]^{-1}=\left[T_{i}(-\theta)\right](i=1,2)}
\end{aligned}
$$

For transformed compliance a similar relationship is established:

$$
[\bar{S}]=\left[T_{2}\right]^{-1}[S]\left[T_{1}\right]
$$

From these transformations it is possible to obtain the elastic properties in any direction. For example, the off-axis elastic modulus is given by

$$
E_{x}=\frac{E_{1}}{m^{4}+m^{2} n^{2}\left(-2 v_{12}+E_{1} / G_{12}\right)+n^{4} E_{1} / E_{2}}
$$

the Poisson's ratio by

$$
v_{x y}=-\frac{\bar{S}_{12}}{\bar{S}_{11}}=\frac{\left(n^{4}+m^{4}\right) v_{12}-m^{2} n^{2}\left(1+E_{1} / E_{2}-E_{1} / G_{12}\right)}{m^{4}+m^{2} n^{2}\left(-2 v_{12}+E_{1} / G_{12}\right)+n^{4} E_{1} / E_{2}}
$$

and the shear modulus by

$$
G_{x y}=-\frac{1}{\bar{S}_{66}}=\frac{E_{1}}{4 m^{4} n^{2}\left(1+2 v_{12}+E_{1} / E_{2}\right)+\left(n^{2}-m^{2}\right)^{2} E_{1} / G_{12}}
$$

The determination of the in-plane elastic properties involves four values $E_{1}, E_{2}, v_{12}$ and $G_{12}$. The measurement of longitudinal and transverse moduli and Poisson's ratio $\left(E_{1}, E_{2}, v_{12}\right)$ is made using tensile coupons oriented at $0^{\circ}$ and $90^{\circ}$. These tests are quite straightforward to perform and to analyse when measuring the elastic properties. For the in-plane shear modulus $\left(G_{12}\right)$ off-axis, $\pm 45^{\circ}$ and Iosipescu test coupons are used, but these tests are not so straightforward to analyse since a complex stress/strain state is induced in the coupons; further details on this subject are well documented in the literature [33-37]. 
Using experimental data from three different unidirectional composites, a comparison is made between the theory and measured values from offaxis tensile tests. Table 11.1 presents the in-plane elastic properties for unidirectional composites [14].

The comparisons are made using the four in-plane elastic properties $\left(E_{1}\right.$, $\left.E_{2}, v_{12}, G_{12}\right)$ and the relationships throughout given by Equations (11.76(11.78) for different off-axis angles. The results are plotted throughout Figs 11.27-11.35.

The theory compares quite well with experimental data, as should be expected. The experimental values for the off-axis shear modulus $G_{x y}$ were computed from the respective $G_{12}$ measured for each off-axis angle. The deviations found are related to experimental errors, which are much more critical and pronounced in the case of the off-axis tensile tests. However, the measurement of the in-plane shear modulus requires the use of off-axis specimens. Although consistent values of in-plane shear modulus may be obtained, specimen geometry must be optimized to reduce the errors provoked by the end-constraint effect [34].

Table 11.1 In-plane elastic properties of unidirectional composites

\begin{tabular}{llllll}
\hline Unidirectional composite & $E_{1}$ & $E_{2}$ & $v_{12}$ & $G_{12}$ & $\begin{array}{l}\text { Fibre } \\
\text { volume } \\
\text { fraction }\end{array}$ \\
\cline { 2 - 6 } & (GPa) & (GPa) & & (GPa) & \\
\hline Aramid/epoxy & 61.1 & 4.1 & 0.435 & 1.48 & 0.55 \\
Carbon/epoxy (T300/5208) & 136.5 & 9.8 & 0.350 & 5.65 & 0.62 \\
Carbon/polyimide (Celion 6000/PMR-15) & 136.5 & 9.8 & 0.350 & 4.92 & 0.57 \\
\hline
\end{tabular}

Source: Herakovich [14].

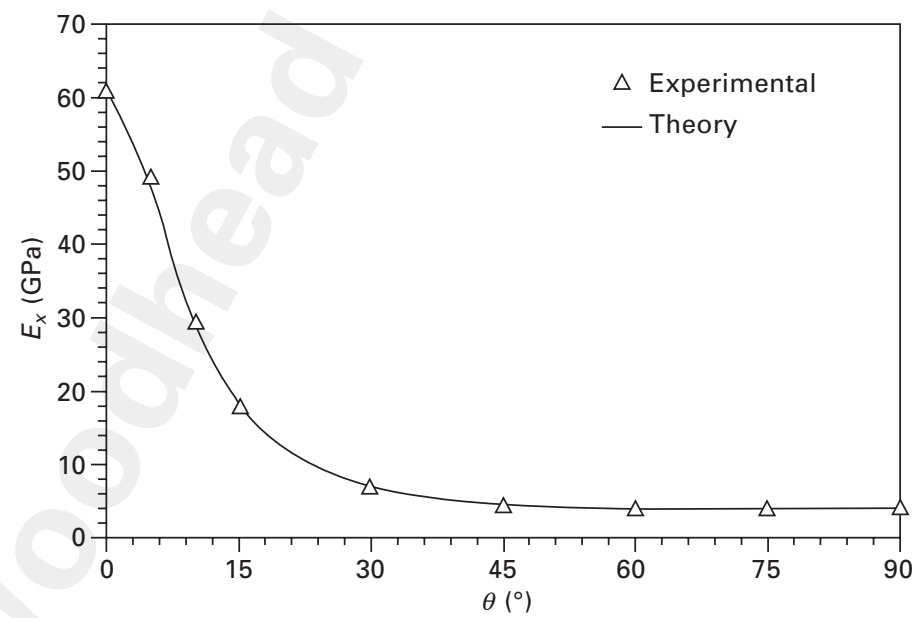

11.27 Off-axis modulus for aramid/epoxy (after [14]). 


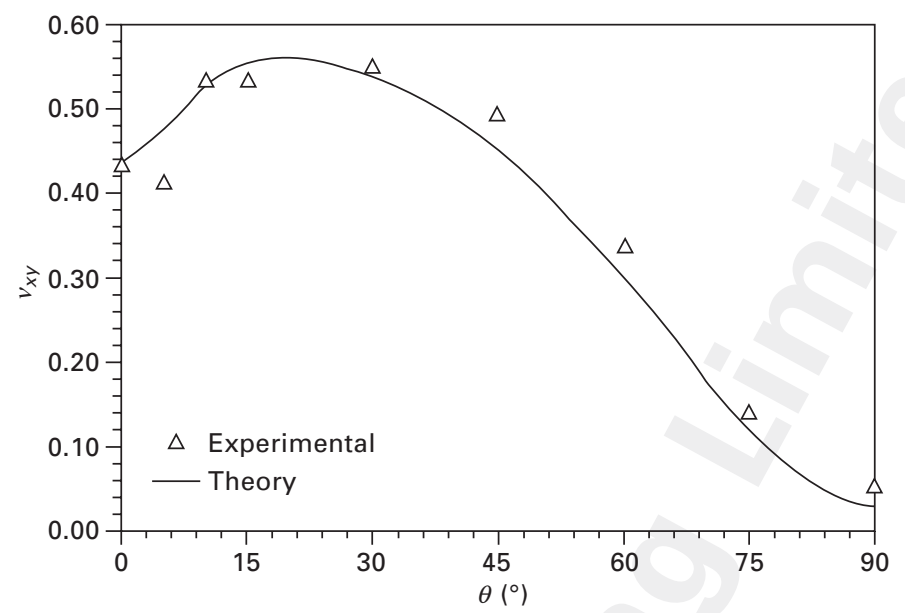

11.28 Off-axis Poisson's ratio for aramid/epoxy (after [14]).

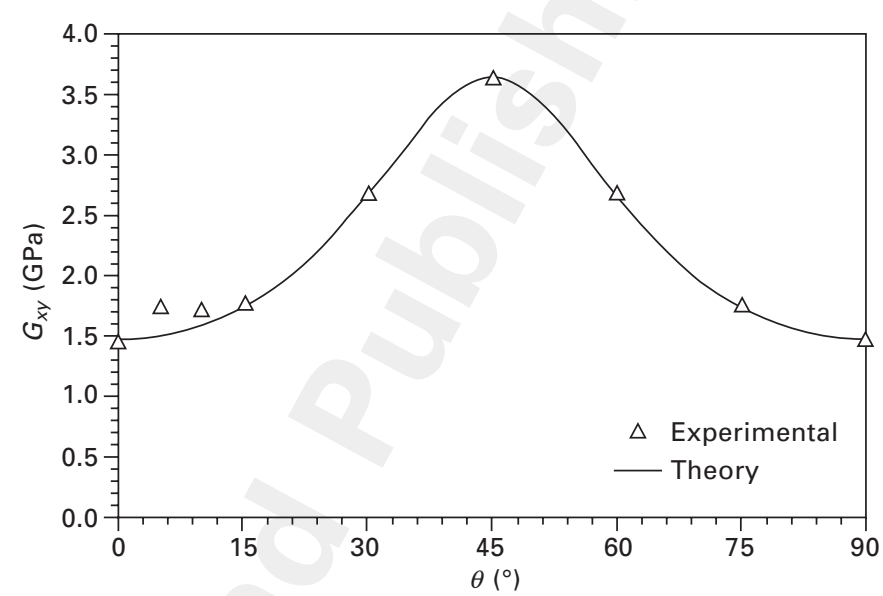

11.29 Off-axis in-plane shear modulus for aramid/epoxy.

\subsection{Laminate plate and shell stiffness: classical lamination theory (CLT)}

Many composite structures can be described and analysed as thin laminated shells or plates composed of several laminae stacked sequentially, each aligned at a specific angle with respect to a material reference axis, by convention the $x$-axis. Classical lamination theory is quite suitable for analysing thin laminated plates or any thin laminated shell that can be reduced to an equivalent plate. 


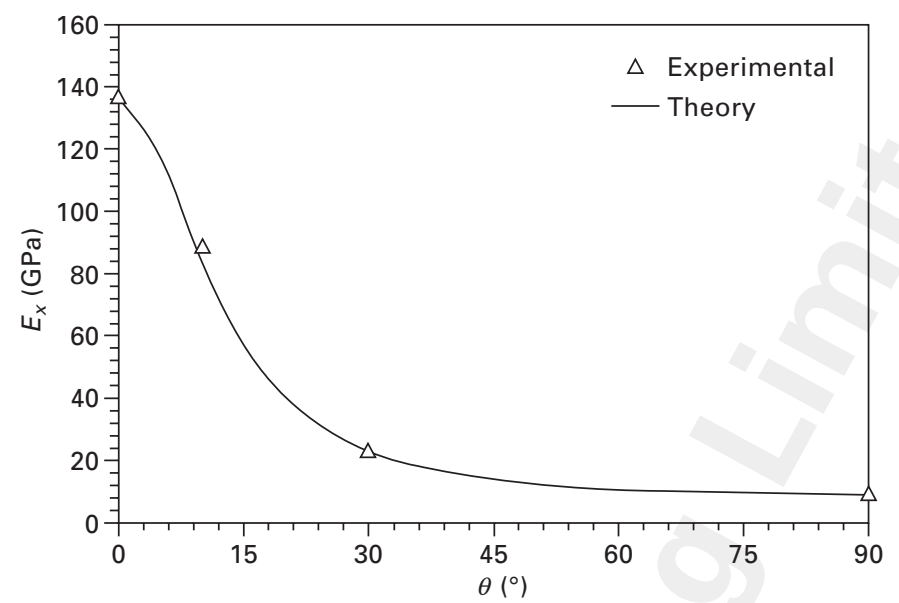

11.30 Off-axis modulus for carbon/epoxy (after [14]).

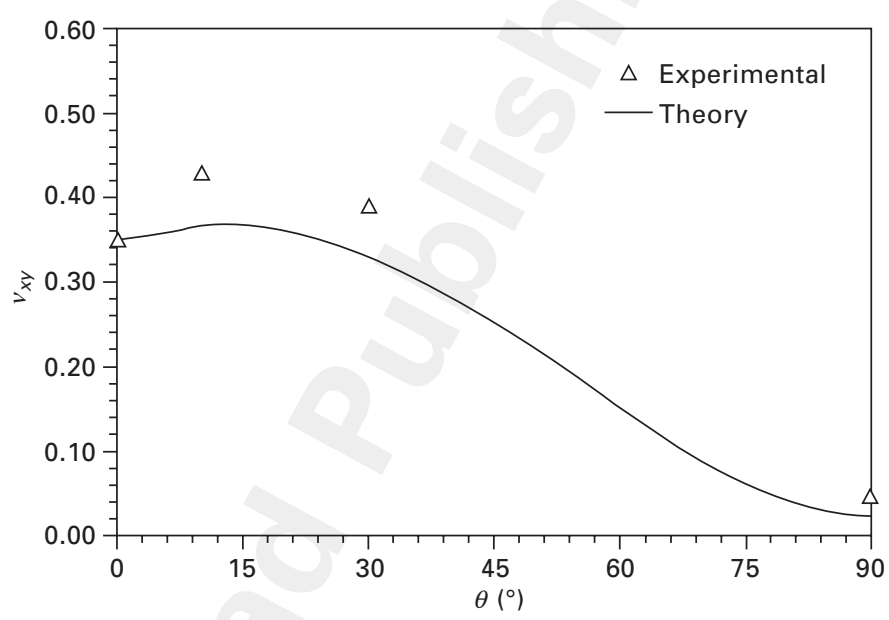

11.31 Off-axis Poisson's ratio for carbon/epoxy (after [14]).

\subsubsection{Laminate code}

The elastic properties of a laminate depend on elastic moduli, stacking position, thickness and angle of orientation of each lamina [14, 15, 38, 39]. Figure 11.36 gives a schematic of the $[0 /+\theta / 90 /-\theta / 0]$ laminate. The code represents each lamina by the angle separated from other plies by a slash sign. The code also implies that each ply is made of the same material and is of the same thickness. For hybrid composites, letters are used after the ply angle to distinguish different materials. The first ply is the top ply of the laminate. 


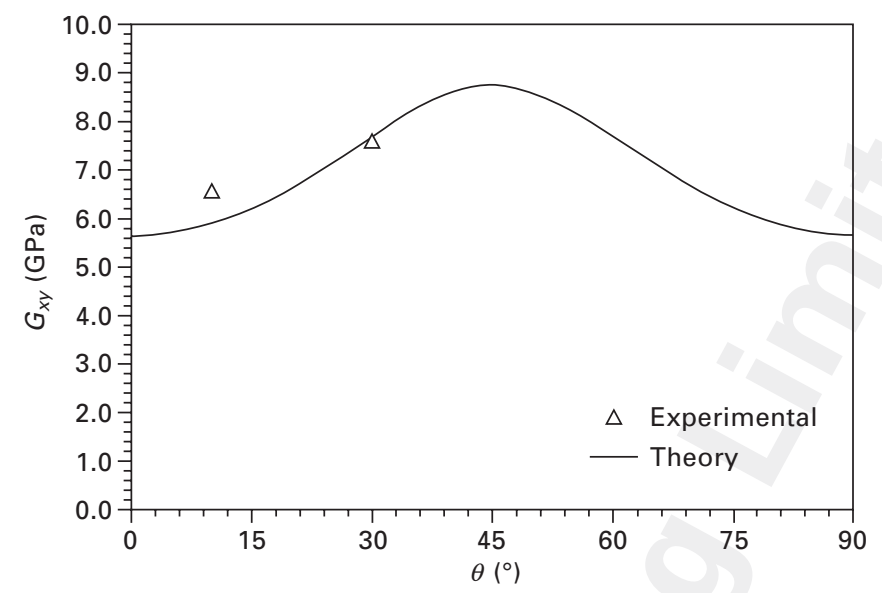

11.32 Off-axis in-plane shear modulus for carbon/epoxy.

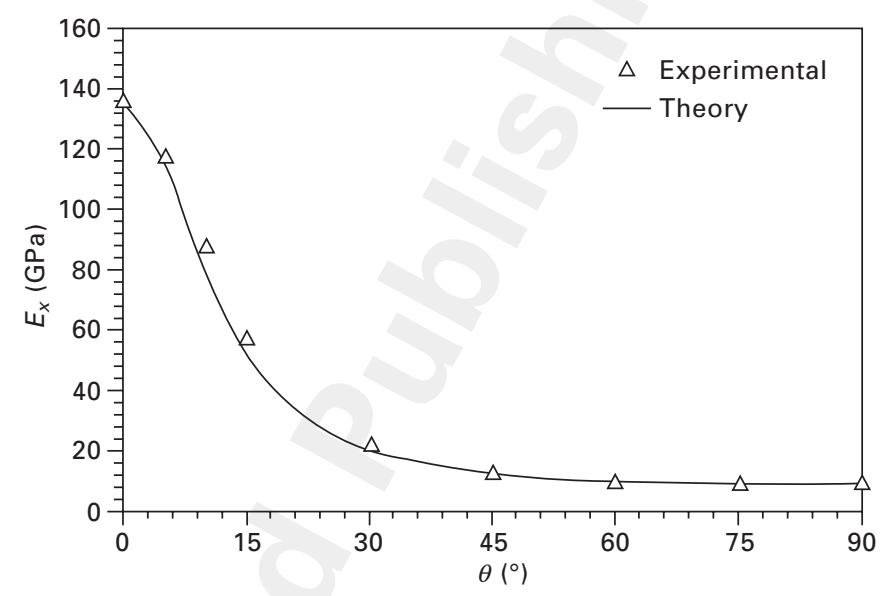

11.33 Off-axis modulus for carbon/polyimide (after [14]).

Special notations are used for symmetric laminates, laminates with adjacent laminae of the same orientation or of opposite angles, and so on.

Figure 11.37 shows four examples of usage of the special notation. The subscript $\mathrm{T}$ outside the brackets stands for total laminate, and the subscript $\mathrm{S}$ stands for symmetry, implying that the total laminate, i.e. the plies in brackets, are repeated in the reverse order. When the number of plies is odd and symmetry exists at the mid-surface, the last ply does not repeat in reverse order and is denoted with a bar on the top. A notation of $\pm \theta$ indicates that the $+\theta^{\circ}$ angle ply is followed by a $-\theta^{\circ}$ angle ply. 


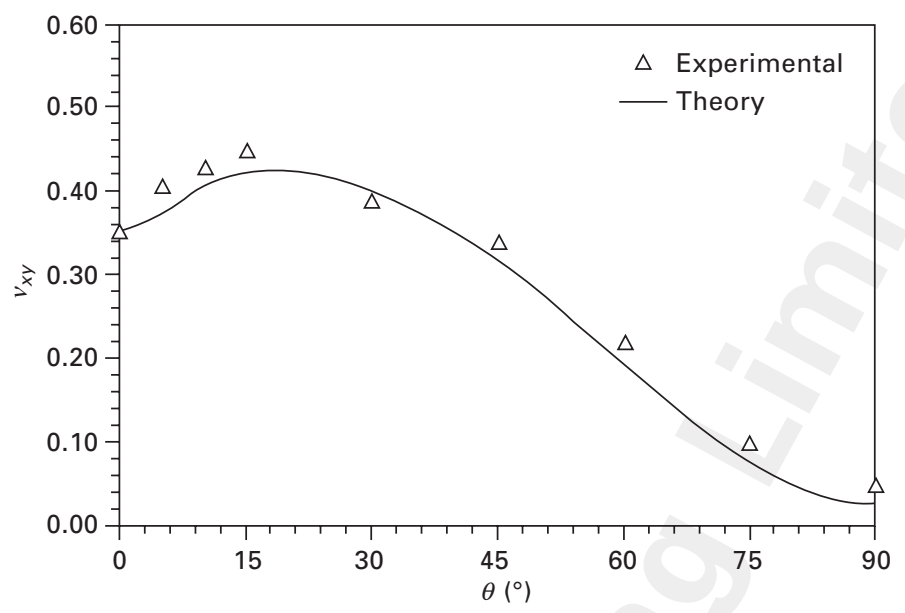

11.34 Off-axis Poisson's ratio for carbon/polyimide (after [14]).

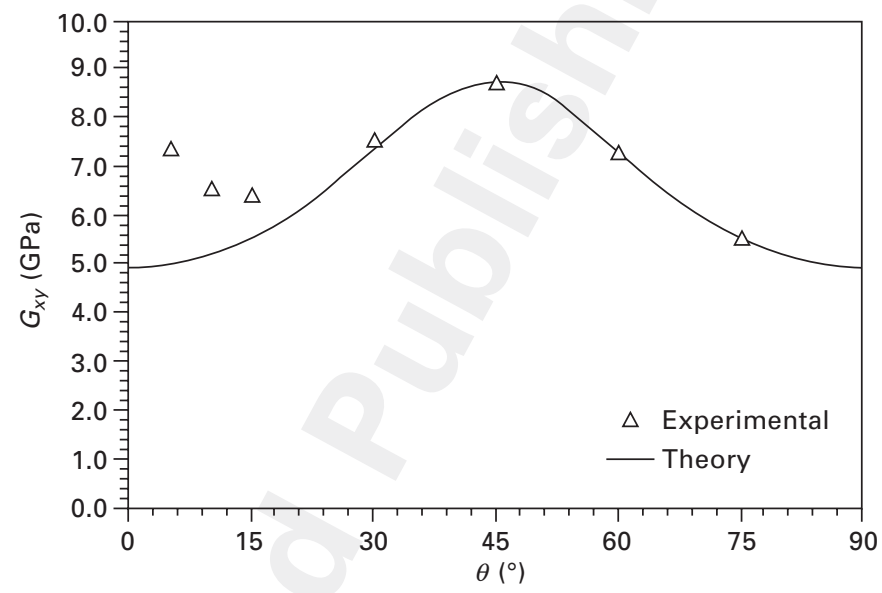

11.35 Off-axis in-plane shear modulus for carbon/polyimide.

\subsubsection{Strain-displacement relationships}

For thin plates subjected to small deformations, the Kirchhoff hypotheses for plates or the Kirchhoff-Love hypotheses for thin plates and shells are assumed [40]. These are as follows:

1. Normals to the mid-plane remain straight and normal to the deformed mid-plane after deformation.

2. Normals to the mid-plane do not change length.

These assumptions lead to the following simplifications. Assumption 1 requires that the shear strains $\gamma_{z x}=\gamma_{z y}=0$. Assumption 2 requires that the 


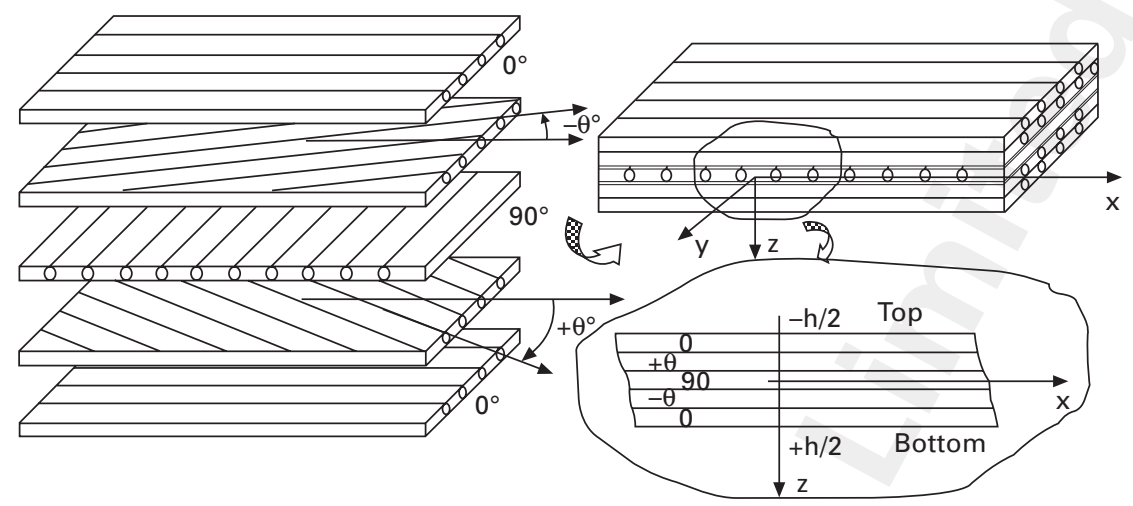

11.36 Schematic of a $[0 /+\theta / 90 /-\theta / 0]$ laminate to illustrate the laminate code.

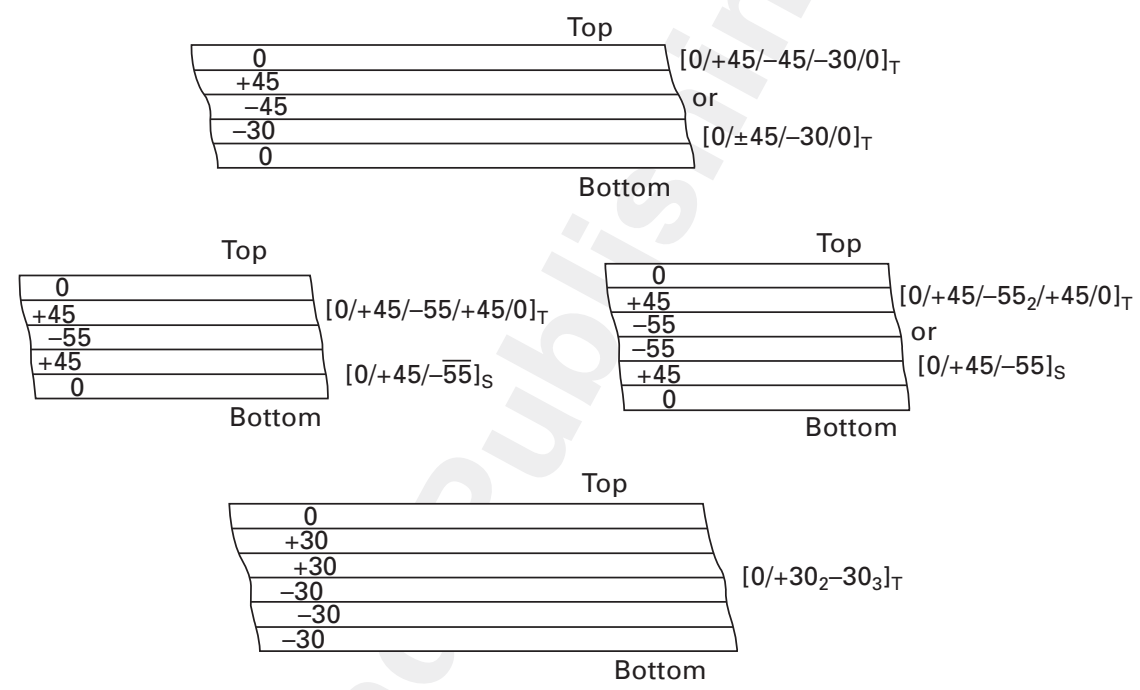

11.37 Examples of the laminate code notation usage.

deflection of the mid-plane depends only on $x$ and $y$ coordinates, i.e. $w=$ $w(x, y)$. Hence this requires that $\varepsilon_{z}=0$.

In Fig. 11.38 the mid-plane deformation is shown schematically. If the displacements are assumed small, the slope, $\tan \alpha$, of the deformed mid-plane will also be small. Since for small angles the approximation $\tan \alpha \cong \alpha$ can be used,

$$
\tan \alpha=\frac{\partial w}{\partial x} \cong \alpha
$$

The total displacement of a generic point in the $x$-axis direction is given by

(C) Woodhead Publishing Limited, 2013 


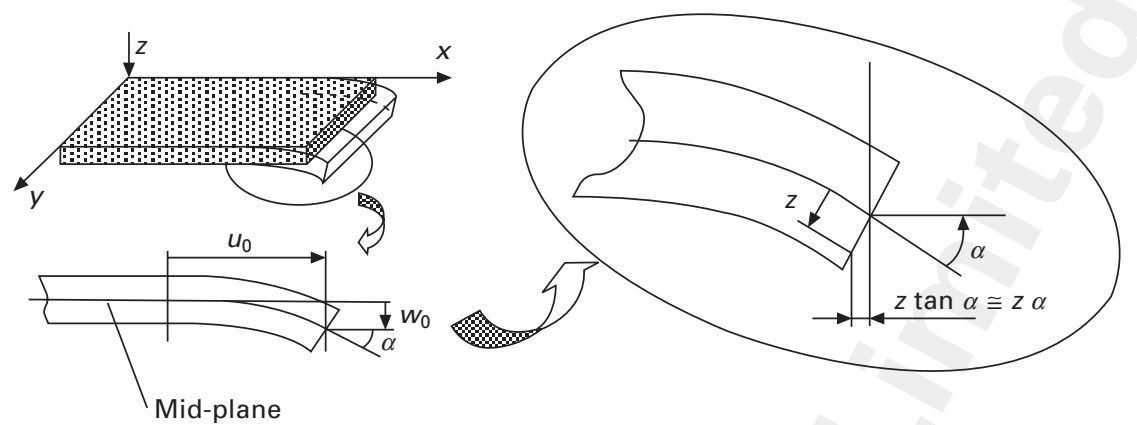

11.38 Schematic description of displacements through the thickness of a plate.

the sum of the mid-plane $u^{0}$ and the displacement produced by the rotation $\alpha$ of the normal to the mid-plane (see Fig. 11.38):

$$
u=u^{0}-z \tan \alpha \cong u^{0}-a \alpha=u^{0}-z \frac{\partial w}{\partial x}
$$

Similarly, the total displacement of a generic point in the $y$-axis direction is given by

$$
v=v^{0}-z \frac{\partial w}{\partial y}
$$

The plane deformations can be readily obtained, from the displacements

$$
\left\{\begin{array}{c}
\varepsilon_{x} \\
\varepsilon_{y} \\
\gamma_{x y}
\end{array}\right\}=\left\{\begin{array}{c}
\frac{\partial u}{\partial x} \\
\frac{\partial v}{\partial y} \\
\frac{\partial u}{\partial y}+\frac{\partial v}{\partial x}
\end{array}\right\}=\left\{\begin{array}{c}
\frac{\partial u^{0}}{\partial x} \\
\frac{\partial v^{0}}{\partial y} \\
\frac{\partial u^{0}}{\partial y}+\frac{\partial v^{0}}{\partial x}
\end{array}\right\}-z\left\{\begin{array}{c}
\frac{\partial^{2} w}{\partial x^{2}} \\
\frac{\partial^{2} w}{\partial y^{2}} \\
2 \frac{\partial^{2} w}{\partial y \partial x}
\end{array}\right\}=\left\{\begin{array}{c}
\varepsilon_{x}^{0} \\
\varepsilon_{y}^{0} \\
\gamma_{x y}^{0}
\end{array}\right\}-z\left\{\begin{array}{c}
\kappa_{x} \\
\kappa_{y} \\
\kappa_{x y}
\end{array}\right\}
$$

where $\varepsilon_{x}^{0}, \varepsilon_{y}^{0}$ and $\gamma_{x y}^{0}$ represent the mid-plane strain state and $\kappa_{x}, \kappa_{y}$ and $\kappa_{x y}$ represent the curvatures.

\subsubsection{Stress-strain relationships and transformation by rotation}

The lamination theory assumes also that each layer is in a state of plane stress. Furthermore it assumes that each lamina is a homogeneous material 
that can be isotropic, transversely isotropic or orthotropic. In the state of plane stress the condition $\sigma_{z}=\gamma_{z x}=\gamma_{z y}=0$ is verified. The stress-strain relationships are devolved from the $3 \mathrm{D}$ relationships to obtain the reduced compliance and stiffness [14]. In the principal material coordinate system, the plane stress-strain relationship is

$$
\left\{\begin{array}{c}
\varepsilon_{1} \\
\varepsilon_{2} \\
\gamma_{12}
\end{array}\right\}=\left[\begin{array}{ccc}
S_{11} & S_{12} & 0 \\
S_{21} & S_{22} & 0 \\
0 & 0 & S_{66}
\end{array}\right]\left\{\begin{array}{c}
\sigma_{1} \\
\sigma_{2} \\
\tau_{12}
\end{array}\right\}
$$

or

$$
\left\{\begin{array}{c}
\varepsilon_{1} \\
\varepsilon_{2} \\
\gamma_{12}
\end{array}\right\}=\left[\begin{array}{ccc}
\frac{1}{E_{1}} & -\frac{v_{21}}{E_{2}} & 0 \\
-\frac{v_{12}}{E_{2}} & \frac{1}{E} & 0 \\
0 & 0 & \frac{1}{G_{12}}
\end{array}\right]\left\{\begin{array}{c}
\sigma_{1} \\
\sigma_{2} \\
\tau_{12}
\end{array}\right\}
$$

where $\frac{v_{21}}{E_{2}}=\frac{v_{12}}{E_{1}}$.

Inverting the former relationships,

$$
\left\{\begin{array}{l}
\sigma_{1} \\
\sigma_{2} \\
\tau_{12}
\end{array}\right\}=\left[\begin{array}{ccc}
Q_{11} & Q_{12} & 0 \\
Q_{21} & Q_{22} & 0 \\
0 & 0 & Q_{66}
\end{array}\right]\left\{\begin{array}{c}
\varepsilon_{1} \\
\varepsilon_{2} \\
\gamma_{12}
\end{array}\right\}
$$

or

$$
\left\{\begin{array}{l}
\sigma_{1} \\
\sigma_{2} \\
\tau_{12}
\end{array}\right\}=\left[\begin{array}{ccc}
\frac{E_{1}}{1-v_{12} v_{21}} & \frac{v_{12} E_{2}}{1-v_{12} v_{21}} & 0 \\
\frac{v_{21} E_{1}}{1-v_{12} v_{21}} & \frac{E_{2}}{1-v_{12} v_{21}} & 0 \\
0 & 0 & G_{12}
\end{array}\right]\left\{\begin{array}{c}
\varepsilon_{1} \\
\varepsilon_{2} \\
\gamma_{12}
\end{array}\right\}
$$

where $\frac{v_{12} E_{2}}{1-v_{12} v_{21}}=\frac{v_{21} E_{1}}{1-v_{12} v_{21}}$.

The 2D transformations for the rotations are obtained readily from the 3D transformations. The stress transformation, by rotation from the principal material coordinates to the global coordinates, is 


$$
\{\sigma\}_{1}=\left[T_{2} \Omega\right]_{x}
$$

where the transformation matrix is

$$
\left\lfloor T_{1}\right\rfloor=\left[\begin{array}{ccc}
m^{2} & n^{2} & 2 m n \\
n^{2} & m^{2} & -2 m n \\
-m n & m n & m^{2}-n^{2}
\end{array}\right] \text { with } m=\cos \theta \text { and } n=\sin \theta
$$

The strain transformation, by rotation from the principal material coordinates to the global coordinates, is

$$
\{\varepsilon\}_{1}=\left[T_{1} Q_{x}\right.
$$

where the transformation matrix is

$$
\left[T_{2}\right]=\left[\begin{array}{ccc}
m^{2} & n^{2} & m n \\
n^{2} & m^{2} & -m n \\
-2 m n & 2 m n & m^{2}-n^{2}
\end{array}\right]
$$

Therefore the transformed stiffness $[\bar{Q}]$ may be determined using the relationships obtained for the stress and strain transformation,

$$
\left.[\bar{Q}]=\left[T_{1}\right]^{-1} L Q\right]\left[T_{2}\right]=\left[\begin{array}{ccc}
\bar{Q}_{11} & \bar{Q}_{12} & \bar{Q}_{16} \\
\bar{Q}_{12} & \bar{Q}_{22} & \bar{Q}_{26} \\
\bar{Q}_{16} & \bar{Q}_{26} & \bar{Q}_{66}
\end{array}\right]
$$

noting that $\left[T_{i}(\theta)\right]^{-1}=\left[T_{i}(-\theta)\right](i=1,2)$.

For transformed compliance a similar relationship is established:

$$
[\bar{S}]=\left[T_{2}\right]^{-1}[S]\left[T_{1}\right]=\left[\begin{array}{lll}
\bar{S}_{11} & \bar{S}_{12} & \bar{S}_{16} \\
\bar{S}_{12} & \bar{S}_{22} & \bar{S}_{26} \\
\bar{S}_{16} & \bar{S}_{26} & \bar{S}_{66}
\end{array}\right]
$$

\subsubsection{In-plane forces and bending moments per unit length}

The in-plane forces or membrane forces and bending moments per unit length, depicted in Fig. 11.39, are defined as through-thickness integrals of planar stresses, assuming that the laminate consists of perfectly bonded laminae or layers.

Using a condensed form, the following is obtained: 


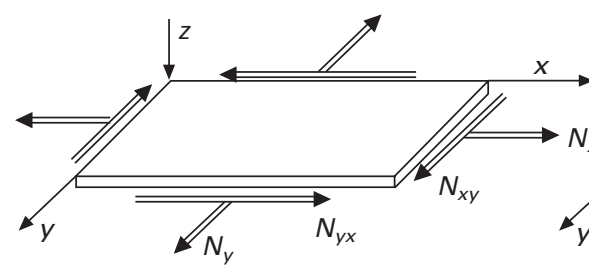

In-plane loads

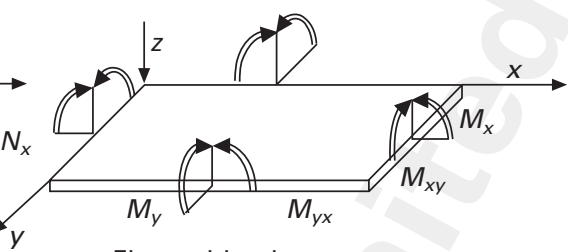

Flexural loads

11.39 Resultant forces and moments on a laminate.

$$
\left\{\begin{array}{l}
N \\
M
\end{array}\right\}=\left\{\begin{array}{l}
\int_{-h / 2}^{+h / 2}\{\sigma\}_{x} \mathrm{~d} z \\
\int_{-h / 2}^{+h / 2}\{\sigma\}_{x} z \mathrm{~d} z
\end{array}\right\}
$$

or

$$
\begin{aligned}
\left\{\begin{array}{l}
N \\
M
\end{array}\right\} & =\left\{\begin{array}{l}
\int_{-h / 2}^{+h / 2}[\bar{Q}]^{k}\left\{\varepsilon^{0}\right\} \mathrm{d} z+\int_{-h / 2}^{+h / 2}[\bar{Q}]^{k}\{\kappa\} \mathrm{zd} z \\
\int_{-h / 2}^{+h / 2}[\bar{Q}]^{k}\left\{\varepsilon^{0}\right\} z \mathrm{~d} z+\int_{-h / 2}^{+h / 2}[\bar{Q}]^{k}\{\kappa\} \mathrm{z}^{2} \mathrm{~d} z
\end{array}\right\} \\
& =\left[\begin{array}{l}
\int_{-h / 2}^{+h / 2}[\bar{Q}]^{k} \mathrm{~d} z \int_{-h / 2}^{+h / 2}[\bar{Q}]^{k} \mathrm{zd} z \\
\int_{-h / 2}^{+h / 2}[\bar{Q}]^{k} z \mathrm{~d} z \int_{-h / 2}^{+h / 2}[\bar{Q}]^{k} \mathrm{z}^{2} \mathrm{~d} z
\end{array}\right]\left\{\begin{array}{l}
\varepsilon^{0} \\
\kappa
\end{array}\right\}
\end{aligned}
$$

where the superscript $k$ stands for the ply number. The integration can be replaced by a summation, since for each ply $[\bar{Q}]^{k}$ is constant. Finally, the fundamental equation of classical lamination theory is obtained:

$$
\left\{\begin{array}{l}
N \\
M
\end{array}\right\}=\left[\begin{array}{ll}
A & B \\
B & D
\end{array}\right]\left\{\begin{array}{l}
\varepsilon^{0} \\
\kappa
\end{array}\right\}
$$

where $N_{\mathrm{c}}$ is the total number of laminae and

$$
\begin{aligned}
& A=\int_{-h / 2}^{+h / 2}[\bar{Q}]^{k} \mathrm{~d} z=\sum_{i=1}^{N_{c}}[\bar{Q}]^{k}\left(z_{k}-z_{k-1}\right) \\
& B=\int_{-h / 2}^{+h / 2}[\bar{Q}]^{k} z \mathrm{~d} z=\frac{1}{2} \sum_{i=1}^{N_{c}}[\bar{Q}]^{k}\left(z_{k}^{2}-z_{k-1}^{2}\right) \\
& D=\int_{-h / 2}^{+h / 2}[\bar{Q}]^{k} z^{2} \mathrm{~d} z=\frac{1}{2} \sum_{i=1}^{N_{c}}[\bar{Q}]^{k}\left(z_{k}^{3}-z_{k-1}^{3}\right)
\end{aligned}
$$


are the submatrices of the laminate stiffness matrix. The laminate stiffness matrix is symmetric. Matrix [A] represents the in-plane stiffness and matrix [D] represents the flexural stiffness. Matrix [B] represents the coupling between in-plane deformations and bending. When the laminate is symmetric, $[B]$ is null, i.e. the in-plane deformations are decoupled from the bending. Matrix [A] does not depend on the stacking sequence, which is not the case for matrix [B] and matrix [D]. When matrix [B] is not null, in-plane loading simultaneously induces in-plane deformations and curvatures. Matrices $[\mathrm{A}],[\mathrm{B}]$ and $[\mathrm{D}]$ are called the extensional, coupling and bending stiffness matrices, respectively. Figure 11.40 gives examples of relationships between in-plane and flexural loads and mechanical behaviour, through the ABD matrix elements.

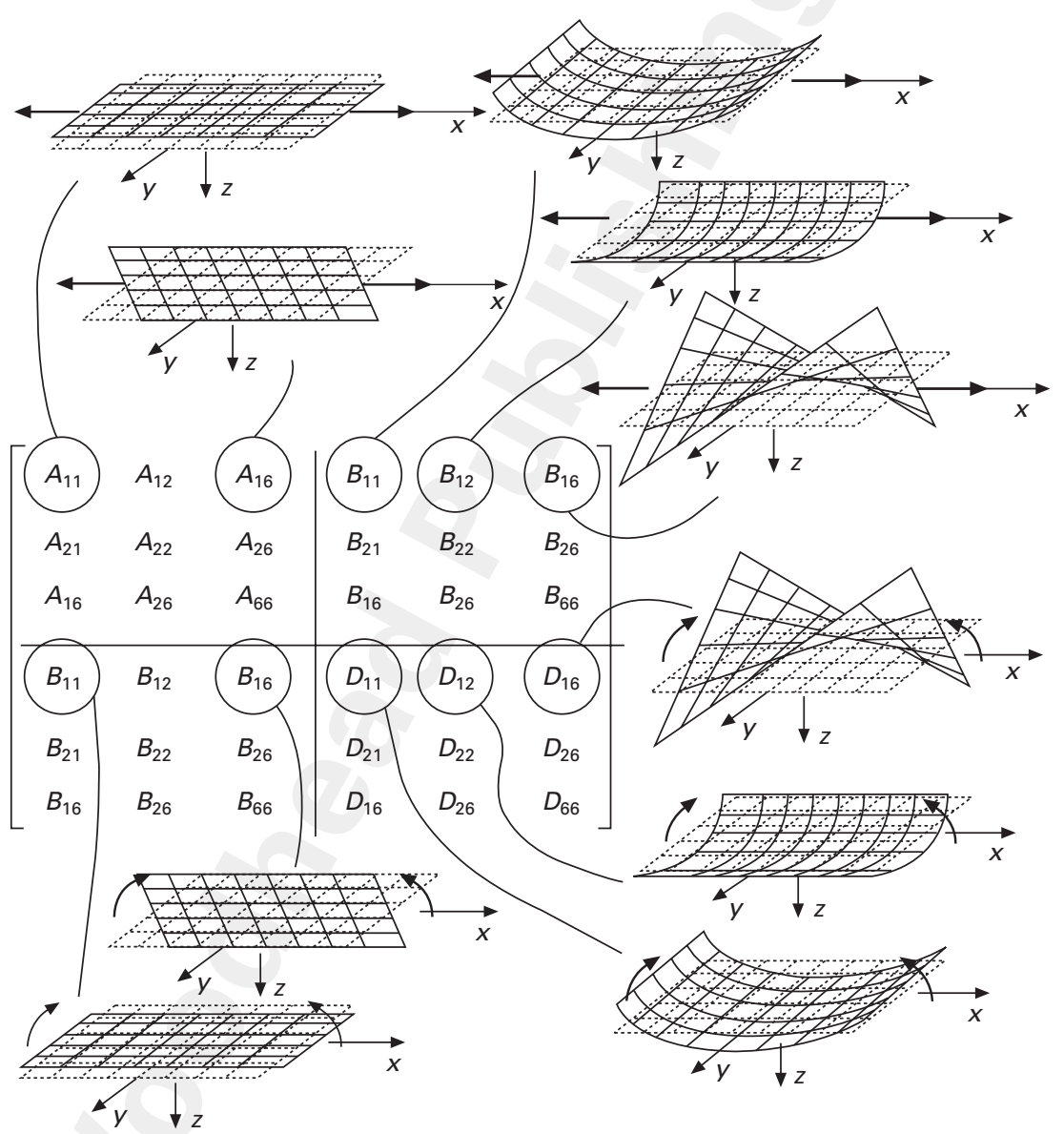

11.40 ABD stiffness matrix and mechanical deformation for in-plane and flexural loading. 
The inversion of Equation (11.93) gives the compliance matrix:

$$
\left\{\begin{array}{l}
\varepsilon^{0} \\
\kappa
\end{array}\right\}=\left[\begin{array}{ll}
A^{\prime} & B^{\prime} \\
B^{\prime T} & D^{\prime}
\end{array}\right]\left\{\begin{array}{l}
N \\
M
\end{array}\right\}
$$

where $\left[A^{\prime}\right]=[A]^{-1}+[A]^{-1}[B]\left[D^{\prime}\right][B][A]^{-1},\left[B^{\prime}\right]=[A]^{-1}[B]\left[D^{\prime}\right],\left[C^{\prime}\right]=\left[D^{\prime}\right][B]$ $[A]^{-1}$ and $\left[D^{\prime}\right]=\left(\left[D^{\prime} \bigcirc B\right][A]^{-1}[B]\right)^{-1}$, also noting that $\left[B^{\prime \mathrm{T}}\right]=\left[B^{\prime}\right]$ and the laminate compliance matrix $A^{\prime} B^{\prime} D^{\prime}$ is symmetric. This comes directly from matrix algebra; the inverse matrix of a symmetric matrix is also symmetric. The $\left[A^{\prime}\right],\left[B^{\prime}\right]$ and $\left[D^{\prime}\right]$ matrices are called the extensional compliance matrix, coupling compliance matrix, and bending compliance matrix, respectively.

After calculating the strains $\left\{\varepsilon^{0}\right\}$ and curvatures $\{\kappa\}$, the stresses can be readily calculated for the $k$ th lamina as

$$
\begin{aligned}
\{\sigma\}^{k} & =[\bar{Q}]^{k}\left\{\varepsilon^{0}\right\}+[\bar{Q}]^{k} z\{\kappa\} \\
& =[\bar{Q}]^{k}\left(\left[A^{\prime}\right]\{N\}+\left[B^{\prime}\right]\{M\}+z\left(\left[B^{\prime}\right]\{N\}+\left[D^{\prime}\right]\{M\}\right)\right)
\end{aligned}
$$

where $z$ represents the distance of the $k$ th lamina centre plane to the laminate mid-plane.

\subsection{Properties of different types of laminate}

\subsubsection{Symmetrical laminates}

In the case of symmetric laminate, the in-plane deformations become uncoupled from bending, i.e. $[B]=\mathbf{0}$. The fundamental equation of classical lamination theory reduces to

$$
\left\{\begin{array}{l}
N \\
M
\end{array}\right\}=\left[\begin{array}{ll}
A & \mathbf{0} \\
\mathbf{0} & D
\end{array}\right]\left\{\begin{array}{l}
\varepsilon^{0} \\
\kappa
\end{array}\right\} \Leftrightarrow\{N\}=[A]\left\{\varepsilon^{0}\right\} \wedge\{M\}=[D]\{\kappa\}
$$

and

$$
\left\{\begin{array}{l}
\varepsilon^{0} \\
\kappa
\end{array}\right\}=\left[\begin{array}{cc}
A^{-1} & \mathbf{0} \\
\mathbf{0} & D^{-1}
\end{array}\right]\left\{\begin{array}{l}
N \\
M
\end{array}\right\} \Leftrightarrow\left\{\varepsilon^{0}\right\}=[A]^{-1}\{N\} \wedge\{\kappa\}=[D]^{-1}\{M\}
$$

\subsubsection{Specially orthotropic laminates}

Laminates with $A_{16}=A_{26}=0$ are designated specially orthotropic because there is no coupling between in-plane extensions and shear deformation. This does not imply necessarily that $D_{16}=D_{26}=0$ because the designation is solely related to the in-plane response. 


\subsubsection{Cross-ply laminates}

Cross-ply laminates are uniquely composed of laminae with fibre orientations of $0^{\circ}$ and $90^{\circ}$. Since for these orientations both $\bar{Q}_{16}=\bar{Q}_{26}=0$, independently of thickness and stacking sequence, for these laminates $A_{16}=A_{26}=0$, i.e. the laminates are specially orthotropic. Moreover, these cases also verify that $D_{16}=D_{26}=0$.

The in-plane stiffness or extensional matrix of a cross-ply laminate of the type $\left[0_{n_{1}} / 90_{n_{2}}\right]_{\mathrm{s}}$, since $\left.\bar{Q}_{11}(0)=\bar{Q}_{22}(90)=Q_{11}, \bar{Q}_{22}(0)=\bar{Q}\right]_{11}(90)=Q_{22}$, $\bar{Q}_{12}(0)=\bar{Q}_{12}(90)=Q_{12}$ and $\bar{Q}_{66}(0)=\bar{Q}_{66}(90)=Q_{66}$, is given as

$$
[A]=2 t\left[\begin{array}{ccc}
n_{1} Q_{11}+n_{2} Q_{22} & \left(n_{1}+n_{2}\right) Q_{12} & 0 \\
\left(n_{1}+n_{2}\right) Q_{12} & n_{2} Q_{11}+n_{1} Q_{22} & 0 \\
0 & 0 & \left(n_{1}+n_{2}\right) Q_{66}
\end{array}\right]
$$

Assuming that all laminae have the same thickness $t$, then the total thickness $h$ is given by $h=2 t\left(n_{1}+n_{2}\right)$. In terms of engineering constants, the equation for $[A]$ is written as

$$
[A]=\frac{2 t}{1-v_{12} v_{21}}\left[\begin{array}{ccc}
n_{1} E_{1}+n_{2} E_{2} & v_{12}\left(n_{1}+n_{2}\right) E_{2} & 0 \\
\left(n_{1}+n_{2}\right) Q_{12} & n_{2} E_{1}+n_{1} Q_{2} & 0 \\
0 & 0 & \left(1-v_{12} v_{21}\right)\left(n_{1}+n_{2}\right) G_{12}
\end{array}\right]
$$

\subsubsection{Angle-ply laminates}

Angle-ply laminates have an equal number of laminae at $+\theta$ and $-\theta$ fibre orientations with equal thickness. It is quite easy to demonstrate that angleply laminates verify $A_{16}=A_{26}=0$, based on the fact that $\bar{Q}_{16}(-\theta)=-\bar{Q}_{16}(\theta)$ and $\bar{Q}_{26}(-\theta)=-\bar{Q}_{26}(\theta)$. Hence angle-ply laminates are specially orthotropic. Contrary to cross-ply laminates, $D_{16} \neq 0 \wedge D_{26} \neq 0$, because the $+\theta$ and $-\theta$ laminae are not at the same distance from the mid-plane. Since $\bar{Q}_{11}(-\theta)=$ $\bar{Q}_{11}(\theta), \bar{Q}_{12}(-\theta)=\bar{Q}_{12}(\theta), \bar{Q}_{22}(-\theta)=\bar{Q}_{22}(\theta)$ and $\bar{Q}_{66}(-\theta)=\bar{Q}_{66}(\theta)$, the in-plane stiffness or extensional matrix can be written as

$$
[A]=h\left[\begin{array}{ccc}
\bar{Q}_{11}(\theta) & \bar{Q}_{12}(\theta) & 0 \\
\bar{Q}_{12}(\theta) & \bar{Q}_{22}(\theta) & 0 \\
0 & 0 & \bar{Q}_{66}(\theta)
\end{array}\right]
$$

where $h$ is the total thickness. 


\subsubsection{Balanced laminates}

In a balanced laminate the laminae with positive angles are balanced by equal laminae with negative angles. Contrary to angle-ply laminates which are restricted to one pair of matched angles, balanced laminates can contain several pairs, including $0^{\circ}$ and $90^{\circ}$. Like angle-ply laminates, for balanced laminates $A_{16}=A_{26}=0$ and $D_{16} \neq 0 \wedge D_{26} \neq 0$.

\subsubsection{Quasi-isotropic laminates}

There is an important group of laminates that exhibit in-plane isotropic elastic response. These laminates are called quasi-isotropic. This group includes all symmetric laminates with $2 N(N>2)$ laminae with the same thickness and $N$ equal angles between fibre orientations $(\Delta \theta=\pi / N)$, i.e. $\Delta \theta=60^{\circ}$ for $N=$ $3, \Delta \theta=45^{\circ}$ for $N=4, \Delta \theta=30^{\circ}$ for $N=6$ and so on. It is possible to prove that the in-plane stiffness or extensional matrix of quasi-isotropic laminates is given in reduced form as [14]

$$
[A]=h\left[\begin{array}{ccc}
U_{1} & U_{4} & 0 \\
U_{4} & U_{1} & 0 \\
0 & 0 & U_{5}
\end{array}\right]
$$

where $h$ is the total thickness and $U_{1}, U_{4}$ and $U_{5}$ are invariants, i.e. only functions of the material, given by [14]

$$
\begin{aligned}
& U_{1}=\frac{3 Q_{11}+3 Q_{22}+2 Q_{12}+4 Q_{66}}{8} \\
& U_{4}=\frac{Q_{11}+Q_{22}+6 Q_{12}-4 Q_{66}}{8} \\
& U_{5}=\frac{Q_{11}+Q_{22}-2 Q_{12}+4 Q_{66}}{8}
\end{aligned}
$$

These laminates are also specially orthotropic since $A_{16}=A_{26}=0$. Hence there is no coupling between in-plane normal and shear responses in these laminates. The designation quasi-isotropic is used because the bending response of these laminates is not isotropic.

\subsection{In-plane and flexural engineering constants of a laminate}

For symmetric laminates it is possible to define effective in-plane moduli in terms of the in-plane stiffness or extensional compliance matrix, since there is no coupling between in-plane and bending response. The effective 
in-plane engineering constants for a laminate with thickness $h$ are readily obtained from the extensional compliance matrix [1],

$$
E_{x}=\frac{1}{h A_{11}^{\prime}}, E_{y}=\frac{1}{h A_{22}^{\prime}}, G_{x y}=\frac{1}{h A_{66}^{\prime}}, v_{x y}=-\frac{A_{12}^{\prime}}{A_{11}^{\prime}}, v_{y x}=-\frac{A_{12}^{\prime}}{A_{22}^{\prime}}
$$

which represent the effective in-plane longitudinal modulus, the effective in-plane transverse modulus, the effective in-plane shear modulus and the two effective in-plane Poisson's ratios, respectively. It should be noted that $v_{x y} / E_{x}=v_{y x} / E_{y}$.

Similarly, the flexural elastic moduli of symmetric laminates are readily obtained from the bending compliance matrix,

$$
E_{x}^{f}=\frac{12}{h^{3} D_{11}^{\prime}}, E_{y}^{f}=\frac{12}{h^{3} D_{22}^{\prime}}, G_{x y}^{f}=\frac{12}{h^{3} D_{66}^{\prime}}, v_{x y}^{f}=-\frac{D_{12}^{\prime}}{D_{11}^{\prime}}, v_{y x}^{f}=-\frac{D_{12}^{\prime}}{D_{22}^{\prime}}
$$

which represent the effective flexural longitudinal modulus, the effective flexural transverse modulus, the effective flexural shear modulus and the two effective flexural Poisson's ratios, respectively. It should also be noted that $v_{x y}^{f} / E_{x}^{f}=v_{y x}^{f} / E_{y}^{f}$.

\subsubsection{Examples}

Examples of angle-ply and quasi-isotropic laminated T300/5208 (carbon/ epoxy) are analysed in terms of in-plane and flexural engineering constants. The lamina engineering constants were given in Table 11.1. Table 11.2 shows the longitudinal modulus and Poisson's ratio for different laminates experimentally measured [14]. The measured data compare quite well with calculated values from CLT.

Table 11.2 Experimental and calculated engineering constants for T300/5208

\begin{tabular}{|c|c|c|c|c|c|c|}
\hline \multirow[t]{2}{*}{ Laminate } & \multicolumn{2}{|c|}{ Experimental } & \multicolumn{4}{|c|}{ Calculated } \\
\hline & $\begin{array}{l}E_{x} \\
\text { (GPa) }\end{array}$ & $v_{x y}$ & $\begin{array}{l}E_{X} \\
(\mathrm{GPa})\end{array}$ & $\begin{array}{l}E_{y} \\
(\mathrm{GPa})\end{array}$ & $v_{x y}$ & $\begin{array}{l}G_{x y} \\
\text { (GPa) }\end{array}$ \\
\hline$\left[10_{2} /-10_{2}\right]_{S}$ & 127 & 0.59 & 126 & 9.92 & 0.672 & 9.1 \\
\hline$\left[ \pm 10_{2}\right]_{S}$ & 123 & 0.56 & 126 & 9.92 & 0.672 & 9.1 \\
\hline$\left[ \pm 30_{2}\right]_{S}$ & 45 & 1.12 & 50.4 & 11.9 & 1.30 & 27.8 \\
\hline$\left[ \pm 45_{2}\right]_{S}$ & 16 & 0.76 & 19.7 & 19.7 & 0.745 & 35.2 \\
\hline$[0 / \pm 45 / 90]_{S}$ & 52 & 0.27 & 53.4 & 53.4 & 0.309 & 20.4 \\
\hline$[90 /-45 / 0 / 45]_{S}$ & 51 & 0.30 & 53.4 & 53.4 & 0.309 & 20.4 \\
\hline
\end{tabular}
laminates

Source: experimental constants are from Herakovich [14] 
Using the angle-ply laminates from Table 11.2, the in-plane and flexural engineering constants, functions of orientation, were calculated and compared through the plots in Figs 11.41-11.43. For all three laminates, $\left[ \pm 10_{2}\right]_{\mathrm{S}},\left[ \pm 30_{2}\right]_{\mathrm{S}}$ and $\left[ \pm 45_{2}\right]_{S}$, it is quite clear that the differences between in-plane and flexural engineering constants are small. The effect of the stacking sequence on the flexural elastic constants is more evident for the $\left[ \pm 30_{2}\right]_{\mathrm{S}}$ laminate. For this laminate the Poisson's ratio becomes negative for directions between $35^{\circ}$ and $55^{\circ}$.

Using the two quasi-isotropic laminates from Table 11.2, the flexural engineering constants, functions of orientation, were calculated and compared

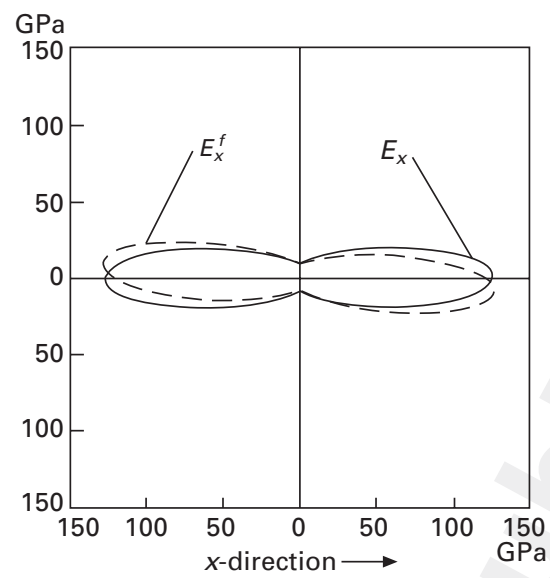

(a)

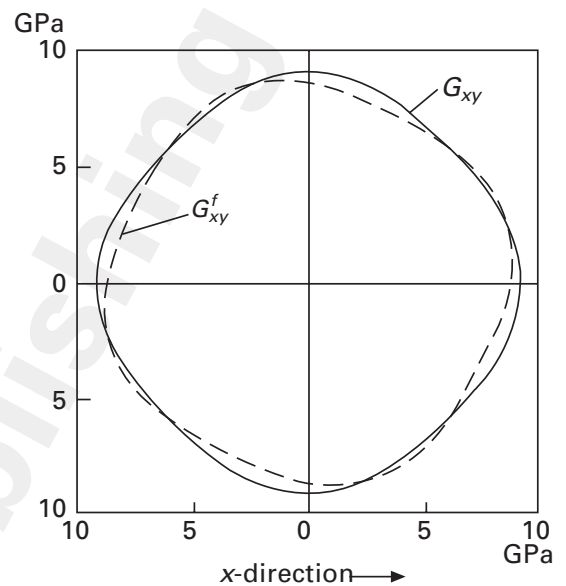

(b)

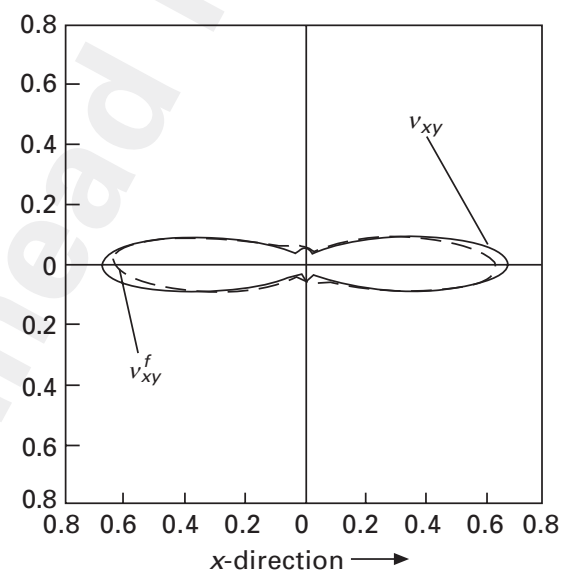

(c)

11.41 Polar variation for the $\left[ \pm 10_{2}\right]_{S}$ laminate of (a) longitudinal modulus, (b) shear modulus and (c) Poisson's ratio. 


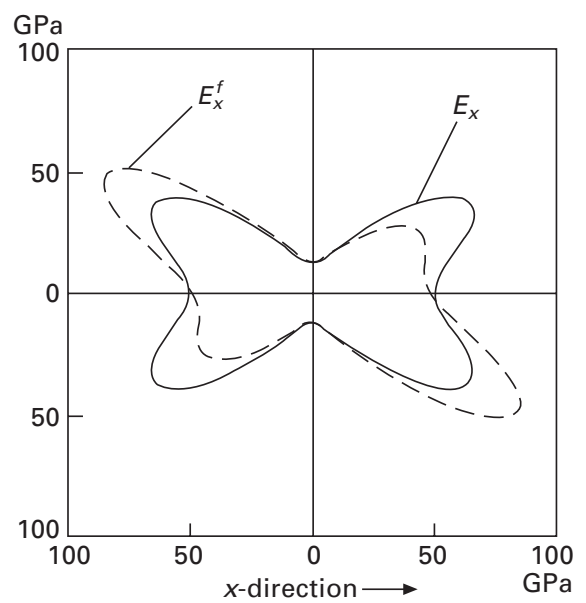

(a)

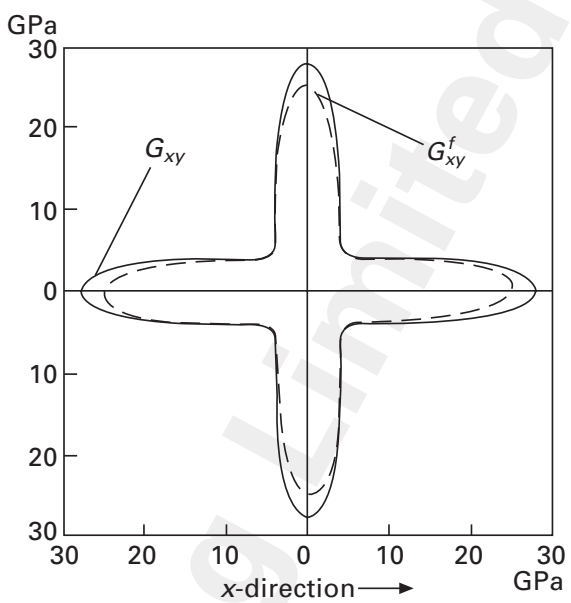

(b)

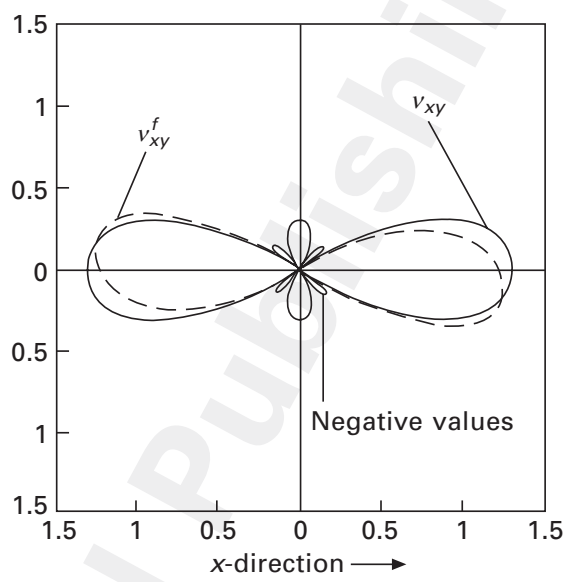

(c)

11.42 Polar variation for the $\left[ \pm 30_{2}\right]_{S}$ laminate of (a) longitudinal modulus, (b) shear modulus and (c) Poisson's ratio.

through the plots in Fig.11.44. It is quite clear that the flexural behaviour is far from being isotropic. The effect of the stacking sequence on the flexural elastic constants is evident from the plots.

The Engineering Sciences Data Unit (ESDU) [41] offers a classification system which uses the ABD stiffness matrices with an extended subscript notation to describe their form, i.e. subscripts 0 if all elements are zero, $\mathrm{F}$ if all elements are not zero (finite), $\mathrm{S}$ if the matrix has the specially orthotropic form and, additionally proposed by York [42], I if it has the isotropic form. Accordingly it is quite easy to describe the ABD stiffness matrix for the following: 


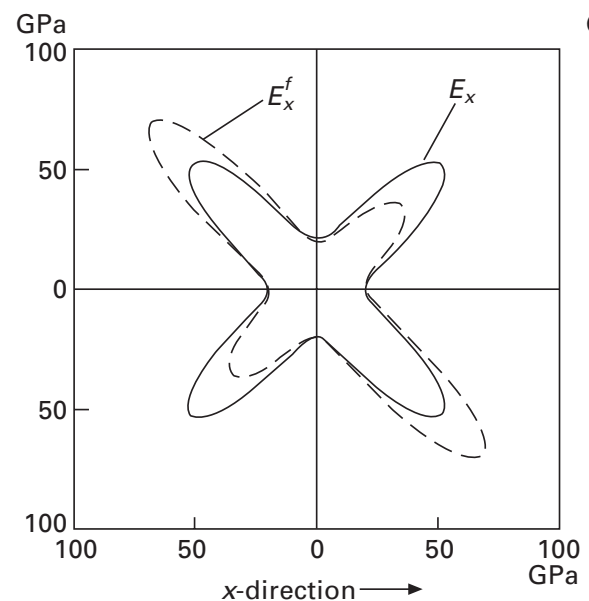

(a)

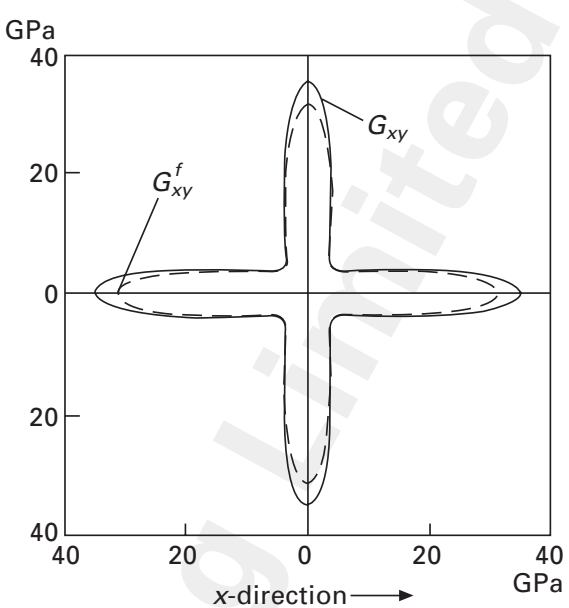

(b)

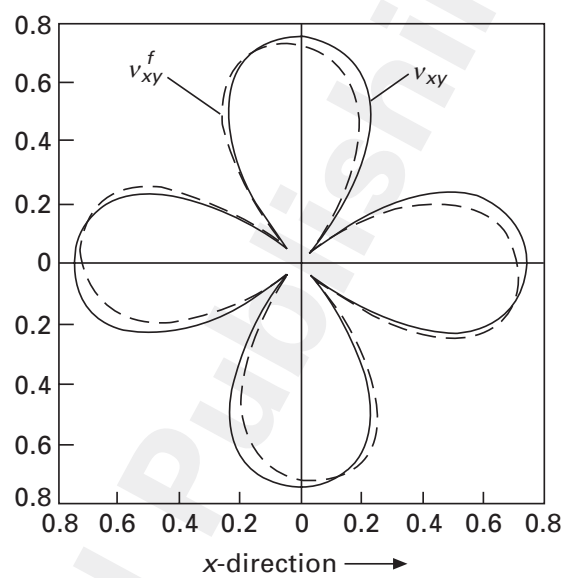

(c)

11.43 Polar variation for the $\left[ \pm 45_{2}\right]_{S}$ laminate of (a) longitudinal modulus, (b) shear modulus and (c) Poisson's ratio.

- Balanced and symmetric laminates: $\mathbf{A}_{\mathrm{S}} \mathbf{B}_{0} \mathbf{D}_{\mathrm{F}}$

- Unbalanced laminates: $\mathbf{A}_{\mathrm{F}} \mathbf{B}_{0} \mathbf{D}_{\mathrm{S}}$

- Fully orthotropic angle-ply laminates: $\mathbf{A}_{\mathrm{S}} \mathbf{B}_{0} \mathbf{D}_{\mathrm{S}}$

- Extensionally isotropic laminates: $\mathbf{A}_{\mathrm{I}} \mathbf{B}_{0} \mathbf{D}_{\mathrm{S}}$

- Fully isotropic laminates: $\mathbf{A}_{\mathrm{I}} \mathbf{B}_{0} \mathbf{D}_{\mathrm{I}}$.

For brevity, the examples given before were restricted to symmetric laminates. However, unsymmetric laminates provide a unique structural behaviour not found in conventional materials. Depending on the prevalent loading conditions and on the specific structural requirements, the selection of the most effective laminate may lead to unsymmetric laminates. 


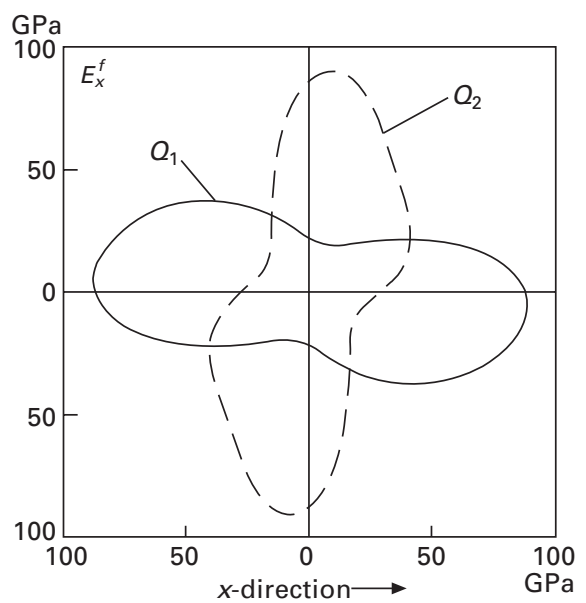

(a)

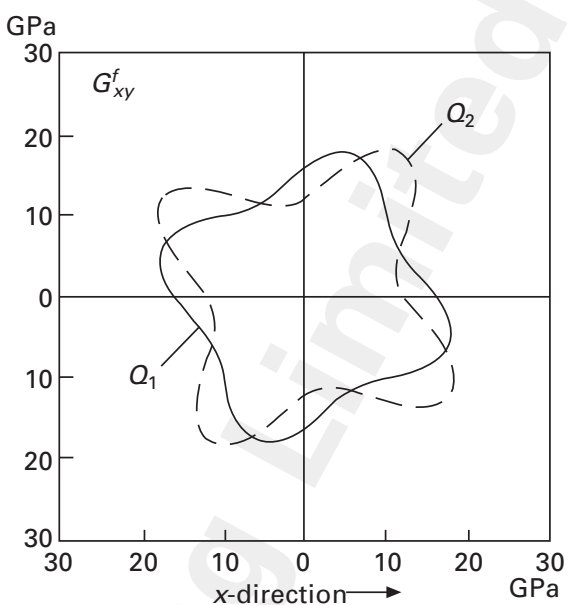

(b)

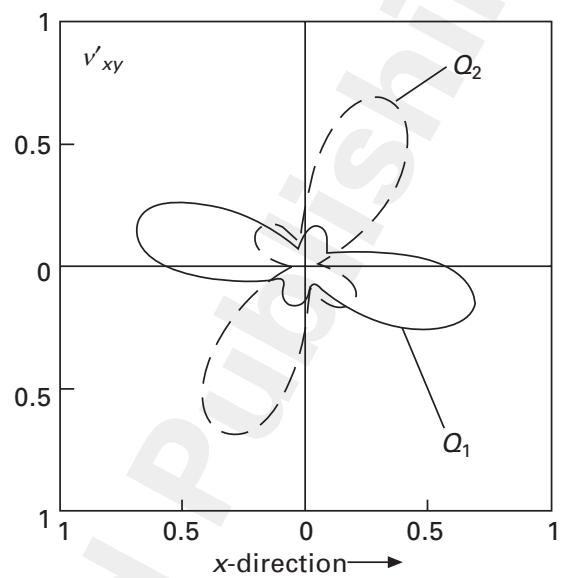

(c)

11.44 Polar variation for the $\mathrm{Q} 1\left([0 / \pm 45 / 90]_{S}\right)$ and $\mathrm{Q} 2\left([90 /-45 / 0 / 45]_{S}\right)$ laminates of (a) flexural longitudinal modulus, (b) flexural shear modulus and (c) flexural Poisson's ratio.

Examples showing that the zero coupling does not require any symmetry were given by Caprino and Crivelli-Visconti [43]. For instance, the uncoupled antisymmetric 8 -ply laminate $[\theta /-\theta /-\theta / \theta /-\theta / \theta / \theta /-\theta]$, with $\theta$ an arbitrary angle, is a fully orthotropic angle-ply laminate, i.e. $\mathbf{A}_{\mathbf{S}} \mathbf{B}_{0} \mathbf{D}_{\mathbf{S}}$. Finally for practical design there is specific software that can be very helpful to assist the CLT calculations. A list is included at the end of this chapter. 


\subsection{New optical methods for measuring laminate stiffness}

Conventionally, experimental mechanics is based on surface deformation measurements (plane stress, plane strain or kinematic assumptions). Mechanical test methods are typically carried out using strain gauges (punctual) or extensometers (average). Data reduction schemes (closed-form solutions) are then proposed for determining material properties as a function of load, specimen dimensions and kinematic (e.g., displacement or strain) measurements. However, in the last decades thanks to the progress of computer science, automated image processing and digital cameras, a photomechanics approach has been emerging. It relies on the use of photonics (the science and technology of generating, controlling and detecting radiant energy) for measuring kinematic quantities (displacement, strain, slope, rotation, etc.) in the branch of mechanics.

Several full-field optical methods have been proposed in solid mechanics [44]. These techniques can be classed according to the physical phenomenon involved in the measurements as white-light (e.g., moiré, grid and digital image correlation methods) and interferometric (e.g., speckle and moiré interferometry, holography and shearography) (Table 11.3). The choice of a given technique can be driven by several criteria such as, for instance, cost, set-up apparatus (simplicity, flexibility, sensitivity to vibrations, etc.), performance (resolution, spatial resolution, etc.), measuring quantity (displacement, strain, etc.), and length scale of observation (from structural

Table 11.3 Optical methods in experimental mechanics

\begin{tabular}{lll}
\hline & \multicolumn{1}{c}{ White-light techniques } \\
\hline Measurand & Periodic pattern & Speckle pattern \\
\hline$u_{x}, u_{y}$ & Geometrical moiré & Digital speckle photography \\
& Grid method & Digital image correlation \\
& Feature tracking method & - \\
$u_{z}$ & Shadow and projection moiré & - \\
& Grid projection & - \\
$u_{x}, u_{y}, u_{z}$ & - & Stereo-correlation \\
$\theta_{x}, \theta_{y}$ & Reflection moiré & - \\
& Deflectometry & - \\
& & Interferometric techniques \\
\hline Measurand & Diffuse light & Diffracted light \\
\hline$u_{x}, u_{y}, u_{z}$ & Speckle interferometry & Moiré interferometry \\
$\varepsilon_{x} \varepsilon_{y}, \varepsilon_{x y}$ & Speckle shearography & Grating shearography \\
\hline
\end{tabular}

Source: after Rastogi [44] and Grédiac [45]. 
down to micro or nano). Contrasting with punctual devices (e.g. strain gauges and extensometers) these methods provide full-field data and are contactfree. The access of this type of information has allowed new insights into different engineering problems, such as, for instance, non-destructive testing, validation of constitutive models and multi-parameter identification from single test configurations [45].

Novel methods have been proposed for material parameter identification, based on full-field measurements (Table 11.4) [46]. This approach has opened new perspectives over classical data reduction schemes. The underlying idea is that a single specimen can be loaded in such a way that several parameters can be simultaneously involved in its mechanical response, yielding complex and heterogeneous stress/strain fields. Providing that these fields are measured by a suitable optical method, the whole set of active parameters can be determined afterwards by a given identification strategy. This approach seems to be particularly convenient for anisotropic and heterogeneous materials.

\subsubsection{Individual optical methods}

In white-light optical techniques the deformation of a material is described by means of the geometrical deformation of a target texture which is assumed to be perfectly attached to the material surface. Moreover, the local luminance of each source point of the imaged pattern is assumed constant throughout the material deformation (optical flow constraint). The analysis is then focused on the spatial variation of the light intensity distribution reflected across the textured pattern. This class of methods can be sorted with regard to periodic (phase-measuring techniques), random (image correlation techniques) or marked (image processing techniques) textures (Table 11.3). A periodic pattern is usually a network of parallel and evenly spaced dark and bright lines, such as the one used in geometrical moiré and grid methods. A random pattern can be imaged by a detector when a rough diffuse surface (roughness $>$ light wavelength) is illuminated by coherent light. In this case, the light is scattered in all directions. Its spatial interference forms a granular pattern, called speckle, which is used as a metrological carrier in digital speckle photography. In digital image correlation, a white-light source is used and the

Table 11.4 Identification methods

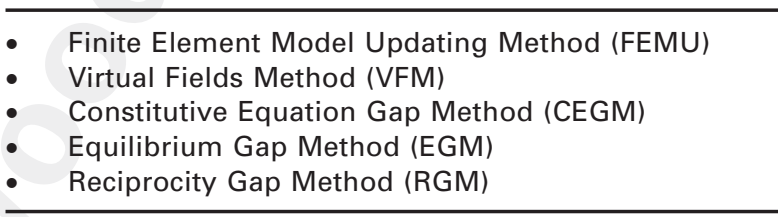

Source: after Avril et al. [46]. 
speckle pattern can be either the natural contrasted texture of the surface of interest or an artificial texture obtained by spraying a black-to-white paint.

An image-based feature tracking method can also be used for measuring displacements. This method requires only a few marks (i.e., local features with suitable colour, shape and size) over the region of interest, which can be transferred (painted) to a background surface. Normally, this technique does not have the same spatial resolution as counterpart DIC and grid methods, but can be suitable for measuring strains over uniform or moderate gradient fields.

Optical interferometry techniques are based on the phenomenon of interference of light waves. These techniques rely on monochromatic and coherent light illumination. Considering the way light interacts with a target surface of interest, these methods can be sorted into diffused light (speckle) and diffracted light (grating) interferometric techniques (Table 11.3). Speckle interferometry is based on the diffuse reflection of light from an optical rough surface, whereas grating interferometry is based on the diffraction of light by a grating attached to the object surface. Several configurations can be set up in which the interference phase variation is given as the dot product of the displacement to the sensitivity vector, given by the difference of illumination and observation wave vectors. Therefore, there are speckle interferometry techniques for both in-plane and out-of-plane displacement measurements. Moiré interferometry is a technique based on grating metrology allowing the measurement of both in-plane and out-of-plane displacements. Finally, there are also different shearography set-ups (Table 11.3) that provide directly the measurement of the (optical) derivatives of the displacement.

Among optical techniques (Table 11.3), digital image correlation (DIC) has become widely used in experimental solid mechanics to study material deformation and fracture mechanics $[47,48]$. On the one hand, with regard to counterpart methods, this technique requires neither specific and expensive optical devices (e.g., lasers and anti-vibration tables) nor time-consuming surface preparation. On the other hand, DIC can be flexibly coupled with mechanical testing machines. Moreover, it can be conveniently applied from the structural to the micro or nano scale. In mechanical tests DIC2D is usually used, whilst in structural applications DIC-3D (stereovision) can be preferable. In contrast with interferometric methods, phase analysis of the fringe images and phase unwrapping process are not required. Nevertheless, DIC measurements are usually unreliable near boundaries or discontinuities, since subsets (continuous shape functions) in the image are typically used for displacement evaluation through mathematical correlation (local approach) $[49,50]$. In the following a review of the DIC method is presented. 


\subsubsection{Digital image correlation}

A schematic representation of the 2D digital image correlation technique coupled with a mechanical test is shown in Fig. 11.45. A (quasi-)planar object is imaged by a camera-lens optical system connected to a computer for realtime visualisation. It is assumed that the surface of interest has a random textured pattern uniquely characterizing the material surface. The magnification of the imaging system is assumed constant during image acquisition (i.e., out-of-plane movements are neglected and the object undergoes only inplane deformation). Besides, geometrical distortions induced by optical aberrations are assumed to be either negligible (differential measurements) or taken into account by a distortion correction method. A matching process is carried out between images taken before and after deformation, as shown schematically in Fig. 11.46. The reference (undeformed) image is divided by square or rectangular subsets defining a given domain ( $\Omega$ in Fig. 11.46). In order to enhance the displacement spatial resolution (defined as the smaller distance separating two independent displacement measurements), subsets can slightly overlap by sharing some pixels. In this case, the subset step $\left(f_{\mathrm{d}}\right)$ will be smaller than the subset size $\left(f_{\mathrm{s}}\right)$. Adjacent $\left(f_{\mathrm{s}}=f_{\mathrm{d}}\right)$ or spaced $\left(f_{\mathrm{s}}\right.$ $<f_{\mathrm{d}}$ ) subsets can also be selected, depending on the purpose. The selection of these measuring parameters is a key issue, because they will contribute to the spatial resolution $(\Delta u)$ and the resolution $\left(\sigma_{u}\right)$ associated with DIC measurements. Therefore, they should be carefully chosen with regard to the application, in a compromise between correlation (small subsets) and interpolation (large subsets) errors. By locally minimizing the difference in light intensity distribution between pairs of images, subset mapping
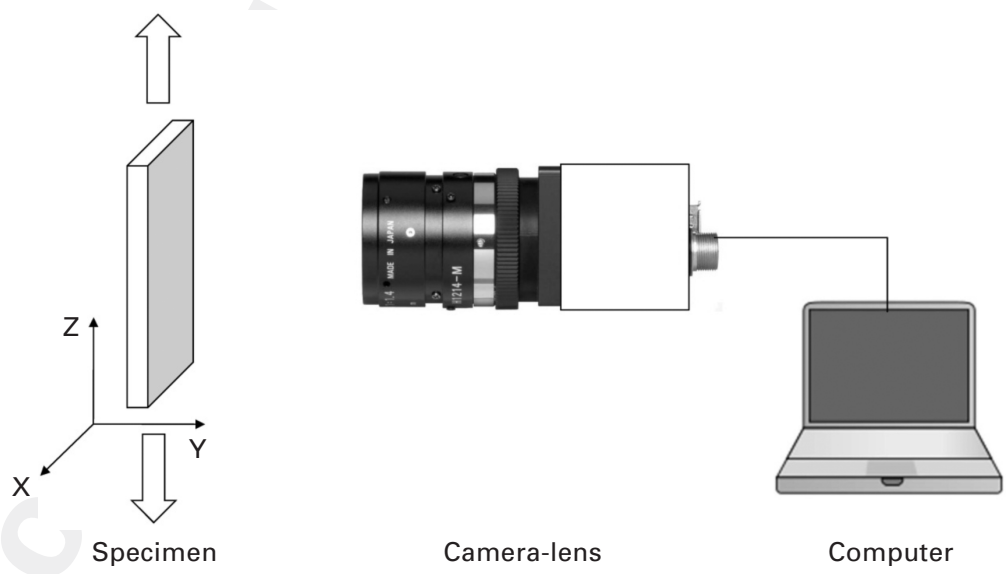

Camera-lens optical system

Computer

11.45 Photo-mechanical setup of the digital image correlation. 


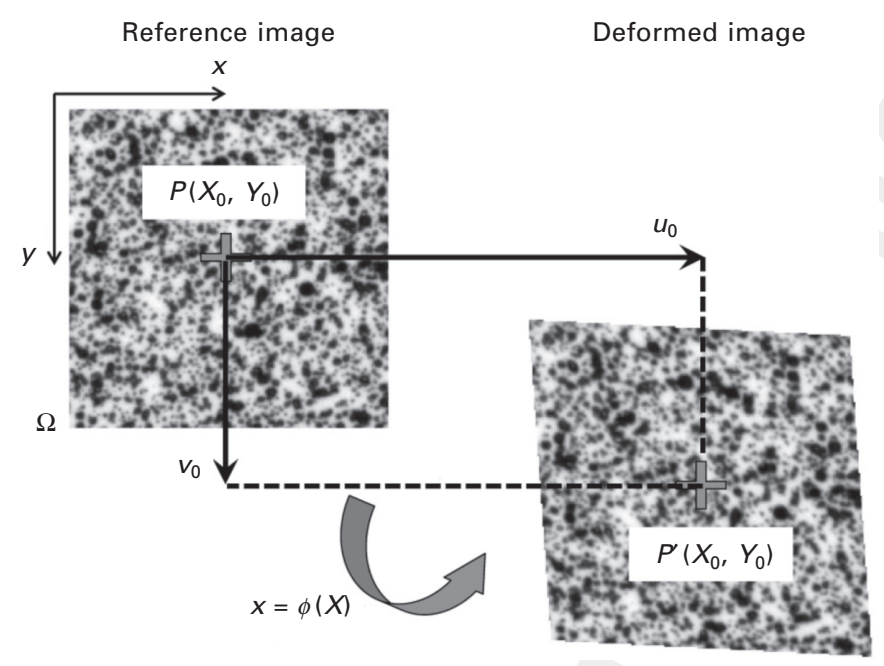

11.46 Schematic representation of the principle of digital image correlation.

in the deformed image is calculated, allowing the definition of full-field displacements.

Several mathematical correlation criteria have been proposed for estimation of the displacement fields in the subset matching process. It has been shown that the zero-normalized sum of squared differences (ZNSSD) is a robust algorithm, since it takes into account offset and linear scale variations of light intensity [48]:

$$
c_{\mathrm{ZNSSD}}(\mathrm{p})=\sum_{\Omega}\left[\frac{f\left(x_{i}, y_{i}\right)-y_{m}}{\sqrt{\sum_{\Omega}}\left(f\left(x_{i}, y_{i}\right)-f_{m}\right)^{2}}-\frac{g\left(x_{i}^{\prime}, y_{i}^{\prime}\right)-g_{m}}{\sqrt{\sum_{\Omega}}\left(g\left(x_{i}^{\prime}, y_{i}^{\prime}\right)-g_{m}\right)^{2}}\right]^{2}
$$

where $\Omega$ is the subset domain, $f\left(x_{i}, y_{i}\right)$ is the pixel grey level at $\left(x_{i}, y_{i}\right)$ in the reference image, $g\left(x_{i}^{\prime}, y_{i}^{\prime}\right)$ is the pixel grey level at $\left(x_{i}^{\prime}, y_{i}^{\prime}\right)$ in the deformed image, and $f_{\mathrm{m}}$ and $g_{m}$ are the mean grey-level values over the subset in the reference and deformed images, respectively.

A given deformation mapping function is to be chosen whose constants define the design parameters (p) to be identified in the matching optimization problem. Both first-order and second-order shape functions have been commonly used [48]. As an example, the first-order transfer is written as:

$$
\left\{\begin{array}{l}
x_{i}^{\prime}-x_{i}=u_{0}+\mathbf{u}_{1}^{\mathrm{T}} \mathbf{d} \\
y_{i}^{\prime}-y_{i}=v_{0}+\mathbf{v}_{1}^{\mathrm{T}} \mathbf{d}
\end{array}\right.
$$

with 


$$
\mathbf{u}_{1}=\left\{\frac{\partial u}{\partial x}, \frac{\partial u}{\partial y}\right\}^{\mathrm{T}}, \mathbf{v}_{1}=\left\{\frac{\partial v}{\partial x}, \frac{\partial v}{\partial y}\right\}^{\mathrm{T}}, \mathbf{d}=\left\{x_{i}-x_{0}, y_{i}-y_{0}\right\}^{\mathrm{T}}
$$

An iterative algorithm, e.g. Newton-Raphson or Levenberg-Marquardt, can then be used for finding the optimal deformation parameter optimizing the correlation coefficient $[48,51]$.

\subsubsection{Identification methods}

The material parameters governing the relevant constitutive equations are determined experimentally through suitable mechanical tests. In the field of solid mechanics, this issue is presented as an inverse problem where the constitutive parameters are to be determined knowing the specimen geometry, the boundary conditions and the strains (or displacements). Conventionally, the identification is carried out on mechanical tests in which geometry and loading conditions yield a homogeneous or simple state of strain/stress across the gauge area. The concept behind this assumption is useful for theoretical analyses because closed-form solutions exist, relating the unknown material parameters to load and strain measurements (statically determined tests). However, the practical implementation of these tests can be difficult, for instance due to end-effects, especially for anisotropic and heterogeneous materials.

The recent development of full-field optical techniques has enabled a new glance on mechanical tests for material characterization [45]. The basic idea underlying this new approach is that a single specimen can be loaded in such a way that several parameters are activated in its mechanical response, yielding heterogeneous and complex strain fields (statically undetermined tests). By means of a suitable identification strategy, all the active parameters can be determined afterwards. A few approaches exist in the literature for addressing this problem (Table 11.4) [46]. The most familiar may be the finite element model updating method (FEMUM). It consists in building a finite element model of the mechanical test and considering a cost function of the difference between numerical and experimental data (displacement or strain) over the region of interest. The minimization of this cost function with respect to the unknown material parameters (design variables), iteratively updated in the model, provides the solution to the problem. This method is flexible and does not specifically require full-field measurements. However, as it is iterative, it can be time-consuming and the convergence can depend on the initial guess of parameters. Moreover, accurate boundary conditions need to be modelled to avoid a bias on the identified parameters. The presence of noise in the measurements will also affect the robustness of the updating routine. To overcome the drawbacks associated with the FEMUM, 
alternative approaches have been proposed, among them the virtual fields method (VFM) [52].

The VFM is based on the fundamental equations of solid mechanics: the equilibrium equation, through the principle of virtual work (PVW), the constitutive equations and the strain-displacement relationships. For an arbitrary solid in equilibrium, the PVW can be written as

$$
\int_{V} \sigma: \varepsilon^{*} \mathrm{~d} V=\int_{S_{\mathrm{f}}} \mathbf{T} \cdot \mathbf{u}^{*} \mathrm{~d} S+\int_{V} \mathbf{f}: \mathbf{u}^{*} \mathrm{~d} V+\int_{V} \rho \gamma \cdot \mathbf{u}^{*} \mathrm{~d} V[11.107]
$$

where $\sigma$ is the stress tensor, $\varepsilon^{*}$ the virtual strain tensor, $V$ the volume of the solid, $\mathbf{T}$ the distribution of external tractions applied over $S_{\mathrm{f}}, \mathbf{u}^{*}$ the virtual displacement, $\mathbf{f}$ the distribution of volume forces acting over $V, \rho$ the mass per unit volume and $\gamma$ the acceleration. Equation (11.107) is valid for any kinematically admissible (K. A.) virtual field (i.e., continuous, differentiable and equal to the prescribed displacements at the displacement boundary conditions). A macroscopic constitutive model of the material must be assumed a priori at this point. In a general case this equation may be written as

$$
\sigma=g(\varepsilon, p)
$$

where $g$ is a given function of the actual strain components $(\varepsilon)$ and of the constitutive parameters $(p)$. By replacing (11.108) into the PVW (11.107) and by neglecting body forces and accelerations, the following relationship is obtained:

$$
\int_{V} g(\varepsilon, p): \varepsilon^{*} d V=\int_{S_{\mathrm{f}}} \mathbf{T} \cdot \mathbf{u}^{*} \mathrm{~d} S
$$

in which the material parameters are related to some kinematic response over a region of interest, geometric characteristics and virtual displacement and strain functions. Generically, the VFM then consists in building up a system of equations by choosing as many independent virtual fields as unknown material parameters. The solution of this system yields the identification of the material properties. In linear elasticity one clear advantage of this method is the direct identification of the unknown constitutive parameters. However, a key issue of the VFM is the selection of such virtual fields yielding to the solution. These functions can be selected either intuitively (a priori choice) or by some automatic process. Polynomial and finite element basis functions have been proposed for automatically generating the virtual fields. Since a large number of possible virtual fields exist, a constrained optimization scheme has also been proposed for selecting virtual fields that minimize the sensitivity of the VFM to noise (maximum likelihood solution). Such strategy was found to significantly improve the robustness of the method.

The design of statistically determined mechanical tests, for which the actual 
state of stress across the gauge area can be deduced directly from the applied load, is most often restricted to simple geometries and loading cases. The main advantage is that simple data reduction can be achieved in these cases. Conversely, more complex tests giving rise to heterogeneous mechanical responses can be carried out for material characterization, providing that full-field measurements are available by a suitable optical method. For that purpose, redesign of mechanical tests is needed. Several test configurations have already been proposed in the literature (Table 11.5) [53]. Most of these tests aim at identifying the linear-elastic properties of orthotropic materials through either in-plane or bending tests. However, some attempts were also made to redesign test methods for identifying elasto-plastic constitutive parameters (Table 11.5). In test design, the configurations must be optimized with regard to the identifiability of the whole set of constitutive parameters to be determined. A general procedure is to build up a cost function that penalizes unbalanced strain components. Generically, this condition may be considered as sufficient to guarantee that several (or all) parameters contribute to the global response of the specimen. Consequently, they can be recovered by a suitable identification strategy afterwards.

\subsection{Conclusion}

This chapter began by describing briefly the elasticity of anisotropic materials, providing the fundamental relationships and the allowed simplifications by the existence of material planes of symmetry. The current unidirectional composites are usually classified as transversely isotropic materials. In this case, only five independent elastic constants are necessary to fully characterize unidirectional composites. The micromechanics provides the analytical and numerical approaches to predict the elastic constants based on the elastic properties of the composite constituents. Several classical closed formulas are revisited and compared with experimental data. Finally, stiffness and compliance transformations are given in the context of unidirectional composites. Experimental data are used to assess theoretical predictions and illustrate the off-axis in-plane elastic properties.

The classical lamination theory was then briefly presented. This is an analytical tool suitable for modelling thin laminated plates in an effective way. Laminate examples were given to illustrate the dependence of in-plane and flexural engineering constants on direction.

The subsequent sections presented an overview of full-field optical techniques and material parameter identification methods. Recent developments in optical methods have allowed a new perspective on the mechanical characterization of advanced composite materials. Several redesigned mechanical tests are summarized, yielding complex and heterogeneous stress/strain fields. By coupling a full-field optical method with a suitable identification strategy, 
Table 11.5 Some heterogeneous mechanical tests for the identification of constitutive parameters

1. Unnotched losipescu test

2. Open-hole tensile test

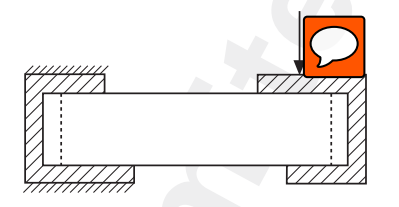

3. Ring compression test

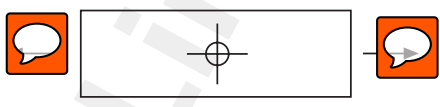

4. Biaxial tensile tests on cruciform specimen

5. Tensile test on flat dog-bone like specimens

6. Bending test on a plate

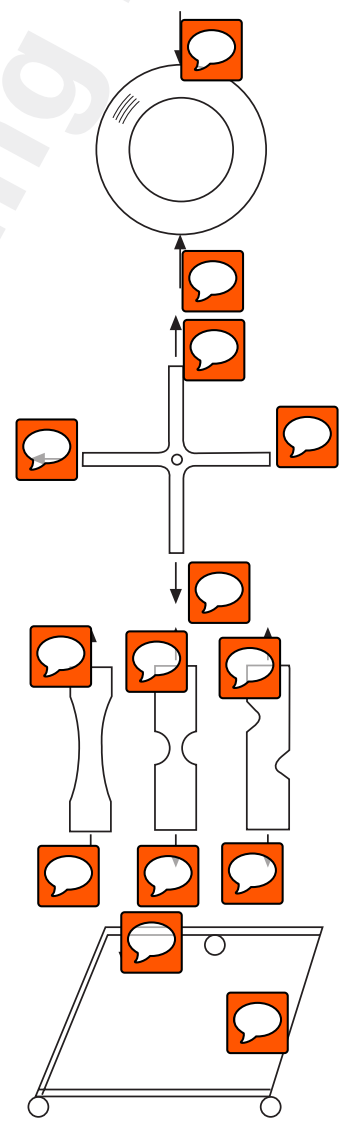

Source: after Xavier [53].

several constitutive parameters can be simultaneously determined on single test configurations. This new approach seems particularly suitable for anisotropic and heterogeneous materials.

(C) Woodhead Publishing Limited, 2013 
This chapter has focused on the elastic qualities of advanced fibre-reinforced composites, in terms of characterization, measurement and prediction from the basic constituents, i.e. the fibre and matrix. Unidirectional fibre-reinforced polymers were the material selected for these brief elastic analyses. These comprised the micromechanics approaches which were applied to predicting the lamina elastic properties from the basic constituents and the classical lamination theory which was used to predict the elastic properties of composites materials composed of several laminae stacked at different orientations. The theoretical predictions were compared against available experimental data, illustrating the predictive capability of the theoretical analysis. Finally, a brief overview was delivered on the identification methods for elastic properties based on full-field measurements. This approach proved to be suitable for anisotropic and heterogeneous materials.

\subsection{Software available}

- Alfalam: Laminate theory, MS Excel, [ABD] matrix, Puck failure criteria, http:/www.klub.tu-darmstadt.de

- CADEC: Computer Aided Design Environment for Composites, Laminate theory, [ABDH] matrix, FEM application, http://www.mae.wvu.edu/ barbero/cadec.html

- eLamX: Laminate theory, Java, [ABD] matrix, 3D failure envelope plots, http://tu-dresden.de

- ESAcomp: Laminate theory, [ABDH] matrix, FEM application http:// www.esacomp.com/

- Genlam: Laminate theory, [ABD] matrix, failure criteria, http://www. thinkcomposites.com/

- LamiCens: Laminate theory, MS Excel, [A] Matrix, Graphics, http:// www.r-g.de.

\subsection{References}

1. Kaw A. K. (2006) Mechanics of Composite Materials, CRC Taylor \& Francis, Boca Raton, FL.

2. Nairn J. A. (2006) in Talreja R. and Manson J.-Å. E., eds Polymer Matrix Composites, Elsevier Science, Amsterdam, the Netherlands, pp. 403-432.

3. Roy S., Xu W., Patel S. and Case S. (2001) International Journal of Solids and Structures, 38, 7627-7641 (2001).

4. Roy S. and Xu W. (2001) International Journal of Solids and Structures, 38, $115-125$.

5. Frost S. R. (2008) Ageing of composite in oil and gas applications, in Martin R., ed. Ageing of Composites, Woodhead Publishing, Cambridge, UK.

6. Roberts S. J., Evans J. T., Gibson A. G. and Frost S. R. (2003) The effect of matrix microcracks on the stress-strain relationship in fibre composite tubes, Journal of Composite Materials, 37(17), 1509-1523. 
7. Zang W. and Gudmundson P. (1993) An analytical model for the thermoelastic properties of composite laminates containing transverse matrix cracks, International Journal of Solids and Structures, 30, 3211-3231.

8. Gudmundson P. and Östlund S. (1992) Numerical verification of a procedure for calculation of elastic constants in microcracking composite laminates. Journal of Composite Materials, 26(17), 2480-2492.

9. Guedes R. M. (ed.) (2011) Creep and Fatigue in Polymer Matrix Composites, Woodhead Publishing, Cambridge, UK.

10. Hashin Z., Rosen B. W., Humphreys E. A., Newton C. and Chaterjee S. (1997) Fibre composite analysis and design: composite materials and laminates, Volume I. Report No. DOT/FAA/AR-95/29,Federal Aviation and Administration, William J. Hughes Technical Center, COTR.

11. González C. and LLorca J. (2007). Mechanical behavior of unidirectional fibrereinforced polymers under transverse compression: Microscopic mechanisms and modeling, Composites Science and Technology, 67(13), 2795-2806.

12. Romanowicz M. (2010) Progressive failure analysis of unidirectional fibre-reinforced polymers with inhomogeneous interphase and randomly distributed fibres under transverse tensile loading, Composites - Part A: Applied Science and Manufacturing, 41(12), 1829-1838.

13. Melro A. R., Camanho P. P. and Pinho S. T. (2008) Generation of random distribution of fibres in long-fibre reinforced composites, Composites Science and Technology, 68(9), 2092-2102.

14. Herakovich C. T. (1997) Mechanics of Fibrous Composites, Wiley.

15. Staab G. H. (1999) Laminar Composites, Elsevier.

16. Chamis C. C. (1984) Simplified composite micromechanics equations for hygral, thermal, and mechanical properties, SAMPE, Quarterly, 15(3), 14-23.

17. Spencer A. (1986) The transverse moduli of fibre-composite material. Composites Science and Technology, 27(2), 93-109.

18. Chamis C.C. and Sendeckyj G. P. (1968) Critique on theories predicting thermoelastic properties of fibrous composites, Journal of Composite Materials, 2, 332-358.

19. Mori T. and Tanaka K. (1973) Average stress in matrix and average elastic energy of materials with misfitting iclusions. Acta Metallurgica, 21, 571-574.

20. Benveniste Y. (1987) A new approach to the application of Mori-Tanaka's theory in composite materials, Mechanics of Materials, 6(2), 147-157.

21. Aboudi J. (1991) Mechanics of Composite Materials: A Unified Micromechanical Approach, Elsevier, Amsterdam.

22. Paley M. and Aboudi J. (1992) Micromechanical analysis of composites by the generalized cells model, Mechanics of Materials, 14(2), 127-139.

23. Aboudi J., Pindera M.-J. and Arnold S. M. (2002) High-fidelity generalized method of cells for inelastic periodic multiphase materials, NASA TM-2002-211469.

24. Tsai S. W. (1964) Structural behavior of composite materials, NASA CR-71, July.

25. Halpin J. C. (1969) Effects of environmental factors on composite materials, AFMLTR-67-423.

26. Halpin J. C. and Kardos J. L. (1976) The Halpin-Tsai equations: a review Polymer Engineering and Science, 16(5), 344-352.

27. Morais A. B. D. (2000) Transverse moduli of continuous-fibre-reinforced polymers, Composites Science and Technology, 60(7), 997-1002.

28. Fu S., Hu X. and Yue C. (1998) A new model for the transverse modulus of 
unidirectional fibre composites, Journal of Materials Science, 33(20), 49534960.

29. Nijhof A. H. J. (1990) Ontwerpen in vezelversterkte Kunststoffen, TU Delft, the Nethedands.

30. Huang Z. M. (2000) Unified micromechanical model for the mechanical properties of two constituent composite materials. Part I: Elastic behavior, Journal of Thermoplastic Composite Materials, 13(4), 252-271.

31. Tsai S. W. and Hahn H. T. (1980) Introduction to Composite Materials, Technomic Publishing, Lancaster, PA. pp. 377-431.

32. Kriz R. D. and Stinchcomb W. W. (1979) Elastic moduli of transversely isotropic graphite fibres and their composites - Equations used to calculate the complete set of elastic transversely isotropic properties for unidirectional fibre-reinforced materials having transversely isotropic fibres are experimentally verified by using improved ultrasonic techniques, Experimental Mechanics, 19(2), 41-49.

33. Pindera M.-J. and Herakovich C. T. (1986) Shear characterization of unidirectional composites with the off-axis tension test, Experimental Mechanics, 26(1), 103112.

34. Pindera M., Choksi G., Hidde J. S. and Herakovich C. T. (1987) A methodology for accurate shear characterization of unidirectional composites. Journal of Composite Materials, 21(12), 1164-1184.

35. Tsai C. L. and Daniel I. M. (1990) Determination of in-plane and out-of-plane shear moduli of composite materials, Experimental Mechanics, 30(3), 295-299.

36. Marín J. C., Cañas J., París F. and Morton J. (2002) Determination of $G_{12}$ by means of the off-axis tension test. part I: Review of gripping systems and correction factors, Composites - Part A: Applied Science and Manufacturing, 33(1), 87-100.

37. Marín J. C., Cañas J., París F. and Morton J. (2002) Determination of $G_{12}$ by means of the off-axis tension test. Part II: A self-consistent approach to the application of correction factors, Composites - Part A: Applied Science and Manufacturing, 33(1), 101-111.

38. Jones R. M. (1975), Mechanics of Composite Materials, Hemisphere Publishing, New York.

39. Tsai S. W. (1992) Theory of Composites Design, Think Composites, Dayton, $\mathrm{OH}$.

40. Love A. E. H. (1906) A Treatise on the Mathematical Theory of Elasticity, 2nd edition, Cambridge University Press, Cambridge, UK.

41. ESDU (1994) Stiffnesses of laminated plates. No. 94003, Engineering Sciences Data Unit, London.

42. York C. B. (2010) Unified approach to the characterization of coupled composite laminates: Benchmark configurations and special cases, Journal of Aerospace Engineering, 23(4), 219-242

43. Caprino G. and Crivelli Visconti I. (1982) A note on specially orthotropic laminates, Journal of Composite Materials, 16(5), 395-399.

44. Rastogi P. K. (2000) Photomechanics, Springer-Verlag, Berlin, p. 472.

45. Grédiac M. (2004) The use of full-field measurement methods in composite material characterization: Interest and limitations, Composites-Part A: Applied Science and Manufacturing, 35(7-8), 751-761.

46. Avril S., Bonnet M., Bretelle A. S., Grédiac M., Hild F., Ienny P., Latourte F., Lemosse D., Pagano S., Pagnacco E. and Pierron F. (2008) Experimental Mechanics, 48, 381-402. 
47. Sutton M. A., Orteu J.-J. and Schreier H. (2009) Image correlation for Shape, Motion and Deformation Measurements: Basic Concepts, Theory and Applications, Springer, New York.

48. Pan B., Qian K., Xie H. and Asundi A. (2009) Two-dimensional digital image correlation for in-plane displacement and strain measurement: a review, Measurement Science and Technology, 20(6), 062001.

49. Sousa A. M. R., Xavier J., Morais J. J. L., Filipe V. M. J. and Vaz M. (2011) Processing discontinuous displacement fields by a spatio-temporal derivative technique, Optics and Lasers in Engineering, 49(12), 1402-1412.

50. Xavier J., Sousa A. M. R., Morais J. J. L., Filipe V. M. J. and Vaz M. (2012) Measuring displacement fields by cross-correlation and a differential technique: experimental validation, Optical Engineering, 51, 043602.

51. Pan B., Xie H., Xu B. and Dai F. (2006) Performance of sub-pixel registration algorithms in digital image correlation, Measurement Science and Technology, 17, 1615-1621.

52. Pierron F. and Grédiac M. (2012) The Virtual Fields Method, Springer, New York.

53. Xavier J. (2007) Characterisation of the wood stiffness variability within the stem by the virtual fields method: Application to P. pinaster in the LR plane. Ph.D. Thesis, Arts et Métiers ParisTech, Châlons-en-Champagne, France. 